\title{
Progresses and challenges in the development of high-field magnets based on RE123 coated conductors
}

Carmine Senatore ${ }^{1}$, Matteo Alessandrini ${ }^{2}$, Andrea Lucarelli ${ }^{2}$, Riccardo Tediosi ${ }^{2}$, Davide Uglietti ${ }^{3}$ and Yukikazu Iwasa $^{4}$

${ }^{1}$ Département de Physique de la Matière Condensée (DPMC) and Département de Physique Appliquée (GAP), University of Geneva, Geneva CH-1211, Switzerland

${ }^{2}$ Bruker Biospin AG, Fällanden, Switzerland

${ }^{3}$ EPFL-CRPP, Fusion Technology, 5232 Villigen-PSI, Switzerland

${ }^{4}$ Francis Bitter Magnet Laboratory, Massachusetts Institute of Technology, Cambridge, MA 02139, USA

\begin{abstract}
Recent progresses in the second generation $\mathrm{REBa}_{2} \mathrm{Cu}_{3} \mathrm{O}_{7-\mathrm{x}}(\mathrm{RE} 123)$ coated conductor (CC) have paved a way for the development of superconducting magnets capable of generating fields well above $23.5 \mathrm{~T}$, i.e. the limit of NbTi$\mathrm{Nb}_{3} \mathrm{Sn}$-based magnets. However, the RE123 magnet still poses several fundamental and engineering challenges. In this work we review the state-of-the-art of conductor and magnet technologies. The goal is to illustrate a close synergetic relationship between evolution of high-field magnets and advancement in superconductor technology. The paper is organised in three parts: 1) the basics of RE123 CC fabrication technique, including latest developments to improve conductor performance and production throughput; 2) critical issues and innovative design concepts for the RE123-based magnet; and 3) an overview of noteworthy ongoing magnet projects.
\end{abstract}

\section{Contents}

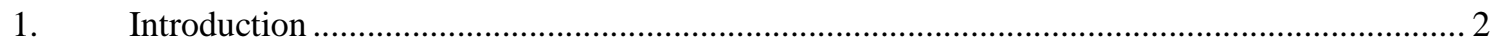

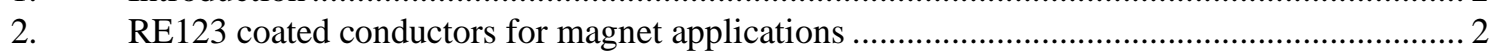

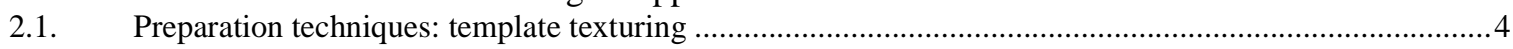

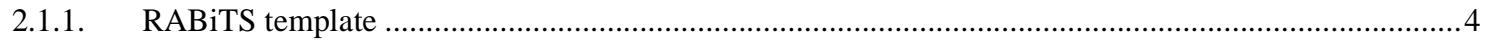

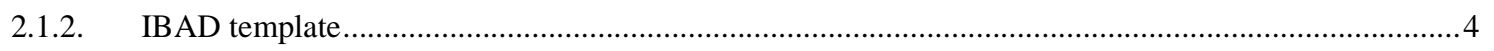

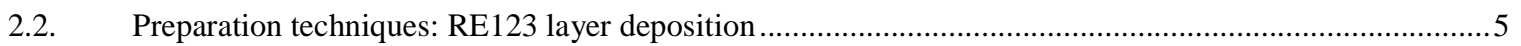

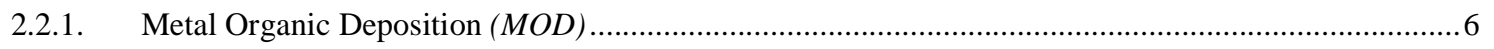

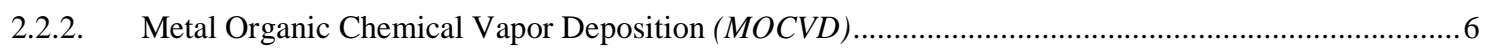

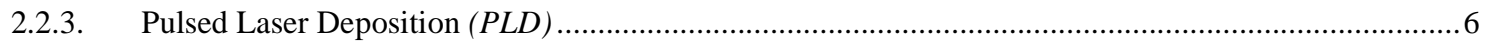

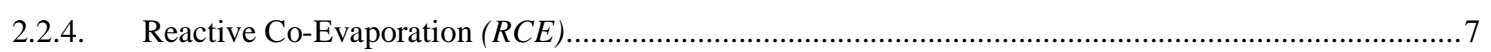

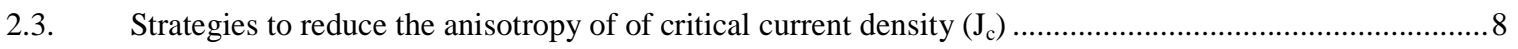

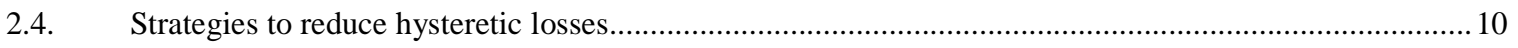

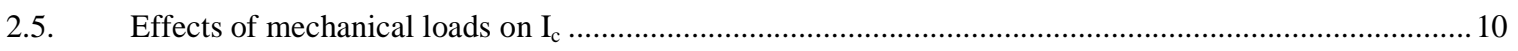


2.5.1. Reversible effects of strain

2.5.2. Irreversible limit under axial loads: influence of the conductor design ....................................... 12

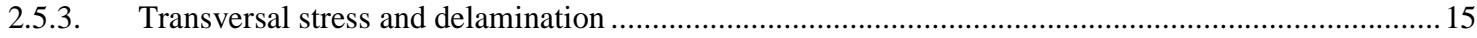

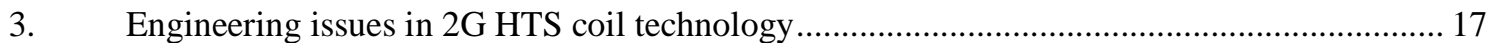

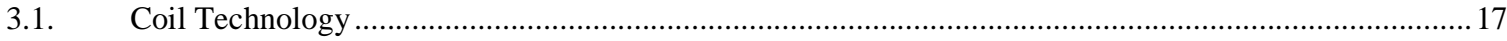

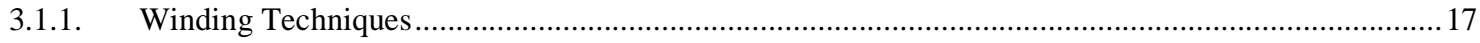

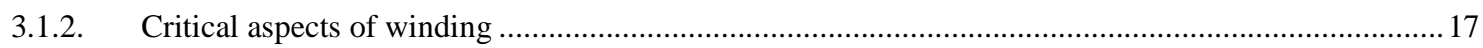

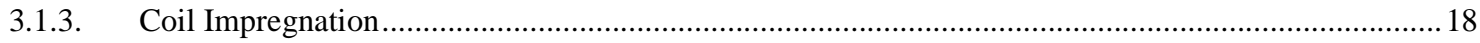

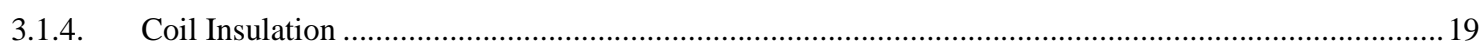

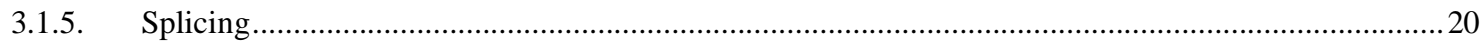

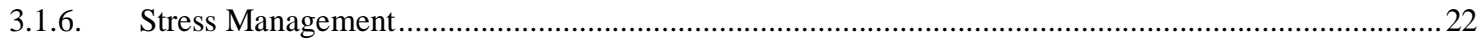

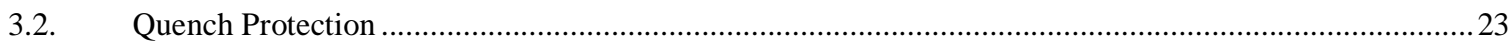

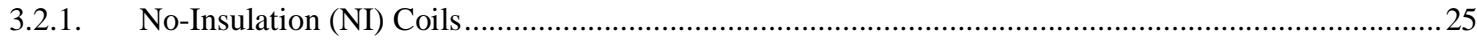

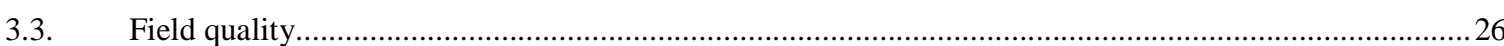

4. Overview on Ongoing High-Field DC Magnet projects based on RE123 ......................... 29

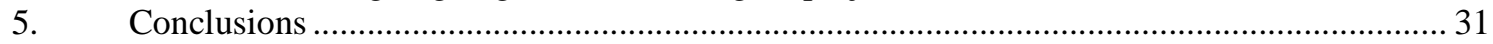

\section{Introduction}

Discovery in 1986 of high-temperature superconductivity (HTS) changed the attitude toward superconductivity of many people, inside science and engineering and even outside. Because for the very first time the remarkable potentials of superconductivity, much touted for so long, appeared much closer to the real world, at least as real as readily accessible liquid nitrogen compared with remote and esoteric world of liquid helium. Although the 77-K barrier was first broken by $\mathrm{YBa}_{2} \mathrm{Cu}_{3} \mathrm{O}_{7-\mathrm{x}}$ (YBCO) in the bulk form [1], YBCO was quickly followed by $(\mathrm{BiPb})_{2} \mathrm{Sr}_{2} \mathrm{Ca}_{n-1} \mathrm{Cu}_{n} \mathrm{O}_{2 n+4}$, known BSCCO that comes in two varieties, Bi2212 (n=2) and Bi2223 (n=3) [2]. From the get-go, BSCCO fabricated in wire became the most eligible HTS for two important applications, power cables and magnets. As have been long established by $\mathrm{NbTi}$ and $\mathrm{Nb}_{3} \mathrm{Sn}$ magnets, the preferred conductor form is indisputably multifilamentary wire. Of the two varieties, only Bi2212 can be fabricated into multifilamentary wire. This is the vital asset of Bi2212 that has kept it relevant from the beginning. For >1-GHz (>23.5 T) NMR magnets, Bi2212, particularly with recent progress [3], is poised to become a viable alternative to the $2^{\text {nd }}$ generation $\mathrm{REBa}_{2} \mathrm{Cu}_{3} \mathrm{O}_{7-\mathrm{x}}$ (RE123, RE = rare earth) coated conductor [4]. Today, RE123 is moving towards a goal of ever-expanding possibilities and opportunities in a three-legged race with its most important partner, the magnet. This paper covers challenges to and recent progress in the RE123 and high-field superconducting magnets. The paper first discusses RE123 issues, including preparation techniques, strategies to reduce critical-current anisotropy, and stress effects on critical current. Most of the second half is devoted on magnet technology, specifically on winding techniques, quench protection, and field quality. The paper ends with an overview of 6 high-field magnets that rely partly or wholly on RE123 coils.

\section{RE123 coated conductors for magnet applications}

In the last ten years the RE123 coated conductors have attracted most of the attention in the applied superconductivity research. The reasons for this excitement are their high current densities in high magnetic fields, 
their outstanding mechanical properties (with Hastelloy or stainless steel as substrate) and, above all, a prospect of economically viable conductor prices.

The development of practical superconductors based on RE123 has revolved around the complexity of achieving high critical current densities in polycrystalline materials. The problem lies in the build up of charge inhomogeneities and strain at the grain boundaries (GBs), regions of mismatch between crystallites with misoriented crystalline axes, that act as weak links that drastically limit the current flow [5]. Therefore, the critical current density through the RE123 GBs falls off exponentially with increasing misorientation angle $\theta$ of adjacent crystallites for $\theta$ above $5^{\circ}-10^{\circ}$ $[5,6]$ : it has been necessary to develop expensive and sophisticated crystallographic texture fabrication processes to eliminate all but low-angle grain boundaries in useful conductor form. A variety of approaches have been implemented at industrial level over the years, but all rely on two common features: a biaxially textured template, consisting of a long and flexible tape-shaped metallic substrate coated with a multifunctional oxide barrier; and an epitaxial RE123 layer. In addition, for environmental protection and thermal stabilization, an Ag layer a few- $\mu \mathrm{m}$ thick and a thicker $\mathrm{Cu}$ layer complete the conductor.

At present, the commercial development of the so-called RE123 coated conductors focuses on three linked issues: performance; price; and production length. On one hand manufacturers aim to improve the conductor performance without increasing the production costs, on the other they are actively pursuing new routes to simplify the wire architecture and increase the produced length and yield, with the goal to reduce the conductor price. The coated conductor cost is mostly due to a large investment in the production infrastructure. The industry is therefore focusing on to process longer lengths of input material in each production run, increase the useful output from the equipment and thus lower the break-even price per unit length. The key ingredients to improve the performance/cost ratio are three:

- Simplify the wire architecture, the production yield being the product of the yield for each production step;

- Perform buffer and RE123 deposition on large areas, followed by a slitting operation to size the conductor to a final width;

- Increase the production line speed.

Kilometer lengths of coated conductors have been successfully demonstrated and lengths of 150+ $\mathrm{m}$ with a minimum critical current of $350 \mathrm{~A} / \mathrm{cm}$-width at $77 \mathrm{~K}$ and in self-field are currently available. However, the present price of $\sim 200 / \mathrm{kA} \mathrm{m}$ (at $\mathrm{B}=2.5 \mathrm{~T}, \mathrm{~T}=30 \mathrm{~K}$ ) [7] is too high for widespread marketplace applications: power device manufacturers (transformers, motors, SMES, cables, fault-current limiters) demand a price less than $\$ 50 / \mathrm{kAm}$.

In a high-field $\mathrm{NbTi} / \mathrm{Nb}_{3} \mathrm{Sn}$ magnet, the operating current is often limited by wire strength (hoop stress), not by critical current. Indeed, for high-field magnets in the field range $19 \mathrm{~T}-24 \mathrm{~T}, \mathrm{RE} 123$ and $\mathrm{Nb}_{3} \mathrm{Sn}$ costs are rather competitive. The reason is mechanical, i.e., an RE123 coil can operate at much higher electromechanical stresses than its LTS counterpart, allowing it to fully exploit available high critical current density. Moreover, in a field above $\sim 18 \mathrm{~T}, \mathrm{Nb}_{3} \mathrm{Sn}$ has to be operated at $2.2 \mathrm{~K}$, while RE123 at $4.2 \mathrm{~K}$. Also note that the tape length required for power equipment is large compared to that for a high-field magnet: hundreds of $\mathrm{km}$ of tape are needed for a $1 \mathrm{~km}-\mathrm{long}$ power cable, whereas continuous lengths of less than $1 \mathrm{~km}$ and total lengths typically of $\sim 10 \mathrm{~km}$ are sufficient for a high-field HTS coil.

The remaining part of the section describes the primary approaches for fabrication of a textured template and deposition of a superconducting layer and addresses the latest developments in the manufacturing process of RE123 coated conductors. 


\subsection{Preparation techniques: template texturing}

In commercial coated conductors, the textured template is created by either deforming the metal substrate with the Rolling Assisted Biaxially Textured Substrate technology (RABiTS) [8] or by texturing the buffer layers by Ion Beam Assisted Deposition (IBAD) [9]. The main features of these two technologies are briefly described below.

\subsubsection{RABiTS template}

In the RABiTS approach, cube texturing is achieved in a NiW substrate through a process of rolling and recrystallization annealing [8]. Compared with pure $\mathrm{Ni}, \mathrm{NiW}$ alloys exhibit reduced magnetization (Curie temperatures of $335 \mathrm{~K}$ for Ni5at. $\% \mathrm{~W}$ and $630 \mathrm{~K}$ for $\mathrm{Ni}$ ) and higher mechanical strength (yield strengths of $\sim 150$ $\mathrm{MPa}$ at room temperature for $\mathrm{Ni5at} \% \mathrm{~W}$ and $\sim 70 \mathrm{MPa}$ for $\mathrm{Ni}$ ).

Ni5at.\%W substrates are industrially prepared in the form of a flexible strip, typically $75-\mu \mathrm{m}$ thick and more than 1$\mathrm{km}$ long. The X-ray full-width half-maximum (FWHM) of the diffraction peak referring to the in-plane texture of the substrate along the entire production length is $\Delta \varphi \sim 6-7^{\circ}[10]$. The out-of-plane $(\Delta \chi)$ FWHM values average approximately $7^{\circ}[10]$. After plasma cleaning to reduce surface roughness, epitaxial buffer layers must be deposited to provide a lattice-matched surface ready for the growth of an HTS layer.

The first layer is typically $\mathrm{Y}_{2} \mathrm{O}_{3}$ (AMSC, [10]) or $\mathrm{CeO}_{2}$ (Sumitomo, [11]). This seed layer achieves an out-of-plane texture that is sharper than the NiW substrate. This is a key step in the production of the RABiTS template. The second yttrium-stabilized zirconia (YSZ) layer behaves as a blocking layer for the inter-diffusion of substrate atoms. Top $\mathrm{CeO}_{2}$ is then prepared to compensate the lattice mismatch between the superconductor and the YSZ layer. The enhanced texture of the seed layer with respect to the substrate propagates throughout the subsequent $\mathrm{YSZ}_{\text {and }} \mathrm{CeO}_{2}$ buffer layers. This template results in an improvement of $\sim 3^{\circ}$ in $\Delta \chi$ and $\sim 1^{\circ}$ in $\Delta \varphi$ [12] compared with the NiW tape. All buffer layers are grown by RF-sputtering. The layer thickness varies among manufacturers, but it is typically of the order of $100 \mathrm{~nm}$.

Further developments of the RABiTS technology are oriented towards substrates characterized by a reduced ferromagnetic behaviour, in order to lower the hysteretic losses especially for power applications. To this end, higher W-content $\mathrm{NiW}$ substrates with the qualified cube texture are being developed [13], but also new preparation approaches for composite substrates are reported [14]. The concept of a composite substrate is to have a thin biaxially textured Ni5at.\%W layer on a non-magnetic Ni9at.\%W base substrate. Although the biaxially textured layer in this clad-type substrate is ferromagnetic, the overall magnetization is reduced.

\subsubsection{IBAD template}

The IBAD technique generates a preferred texture in the buffer layer, hence it does not require a textured substrate. Polycrystalline Hastelloy or stainless steel tapes (typical 50-100 $\mu \mathrm{m}$ thick) are submitted to an electropolishing process. This process reduces surface roughness from $\sim 50 \mathrm{~nm}$ to less than $2 \mathrm{~nm}$ [15]. On the polished substrate surface an $\mathrm{Al}_{2} \mathrm{O}_{3}$ diffusion-barrier and an $\mathrm{Y}_{2} \mathrm{O}_{3}$ seed layers are deposited by sputtering technique. $\mathrm{The}_{2} \mathrm{Al}_{2} \mathrm{O}_{3}$ and $\mathrm{Y}_{2} \mathrm{O}_{3}$ layers are 100-200 nm and 10-20 nm thick, respectively [16]. A biaxially textured $\mathrm{MgO}$ layer is then grown on the $\mathrm{Y}_{2} \mathrm{O}_{3}$ layer with the Ion Beam Assisted Deposition. This technique deposits $\mathrm{MgO}$ in a standard PLD geometry, using a RF plasma source that assists ion beam. The assisting ion is typically $\mathrm{Ar}^{+}$with beam energy of $\sim 1 \mathrm{keV}$ and an irradiating angle between $45^{\circ}$ and $55^{\circ}$ from the substrate normal.

Two mechanisms have been proposed to explain how ion beams affect the crystallographic orientation of growing films. The variation of growth rate with crystal orientation is associated with the existence of crystallographic 
directions along which ions easily penetrate into the crystal. Consequently, grains having these channeling directions aligned parallel with the ion beam have a higher growth rate than nonaligned grains. The second mechanism is related to the recrystallization on the substrate. The aligned grains are less damaged than nonaligned grain by the ions. During the thermal spike associated with ion bombardment, the aligned grains can grow into their more damaged surroundings, thereby reorienting the more damaged material [17]. Typically an appropriate texture is achieved for a very thin layer $(<10 \mathrm{~nm})$, though a homoepitaxial layer of $\mathrm{MgO}(\sim 50 \mathrm{~nm})[15]$ is often added. The typical in-plane texture $\Delta \varphi$ in the $\mathrm{MgO}$ layer is $<10^{\circ}[16]$.

The next process may vary among manufacturers. At Fujikura, a 500-nm thick $\mathrm{CeO}_{2}$ layer is deposited on $\mathrm{MgO}$ and a sharp in-plane texture $\Delta \varphi=4^{\circ}$ is obtained [18]. SuperPower, SuNAM and SuperOx grow a $\mathrm{LaMnO}_{3}$ layer $(20-30$ $\mathrm{nm}$ ) by sputtering atop the homoepitaxial $\mathrm{MgO}[15,19,20]$.

A variant of the IBAD process, the Alternating Beam Assisted Deposition (ABAD), has been developed by Bruker [21]. In this approach, the substrate, first exposed to a molecular flow provided by a sputter source, acquires a few nanometer-thick layer of YSZ. In the following step this layer undergoes an ion etching with an $\mathrm{Ar}^{+}$ion beam. After several tens of deposition-etching cycles, a sufficiently high in-plane texture (with FWHM $=8-9^{\circ}$ ) is achieved in the YSZ layer. A high rate pulsed laser deposition (HR-PLD) is then used for growing a $\sim 0.1-\mu \mathrm{m}$ thick $\mathrm{CeO}_{2}$ cap layer.

The American manufacturer STI has focused on the development of a simplified IBAD architecture where the unpolished Hastelloy substrates are planarized by a solution deposition process (SDP) [22], eliminating the need for an electropolishing step. The idea is to coat the unpolished substrate with a liquid solution: the liquid surface tension planarizes the free surface, resulting in thicker layers over valleys and thinner layers over peaks, overall, a smoother surface than that with the underlying substrate. Specifically, several coatings of $\mathrm{Y}_{2} \mathrm{O}_{3}$ and $\mathrm{Y}_{2} \mathrm{O}_{3}-\mathrm{Al}_{2} \mathrm{O}_{3}$ mixtures are used to planarize the substrate, leading to a surface roughness of less than $\sim 2 \mathrm{~nm}$. SDP has thus the advantage of replacing three steps: electropolishing; barrier; and nucleation layer deposition. The material deposited by SDP is then used as a bed layer for the IBAD and homoepitaxial MgO. In the STI template, HTS is deposited on the MgO layer without the need for additional epitaxial buffers, such as $\mathrm{LaMnO}_{3}$ or $\mathrm{CeO}_{2}$.

It should be stressed that for power applications both NiW and Hastelloy/Steel substrates can be used, while for highfield magnets the substrate mechanical strength of Hastelloy trumps that of NiW. Indeed, this superior mechanical strength makes the Hastelloy-substrate RE123 coated conductor a preferred option to all other HTS for high-field (>20 T) magnets.

\subsection{Preparation techniques: RE123 layer deposition}

In commercial coated conductors multiple techniques for depositing epitaxial RE123 are presently being adopted, with a goal of achieving a low-cost flexible process that is also easily scalable to mass industrial production. Table 1 lists key parameters of the coated conductors among manufacturers. The RE123 layer can be deposited either by chemical routes, such as metal organic deposition (MOD) [23] and metal organic chemical vapor deposition (MOCVD) [24], or by physical routes, such as pulsed laser deposition (PLD) [11, 16, 25, 26] and reactive coevaporation (RCE) [15, 22]. MOD and RCE are ex-situ processes incorporating two steps: deposition of the precursors and subsequent conversion of the precursors into RE123. On the other hand, deposition and formation occur simultaneously during in-situ processes such as PLD and MOCVD.

The basic principles defining these alternative approaches currently used at industrial level are summarized in the following subsections. 


\subsubsection{Metal Organic Deposition (MOD)}

In the MOD process a solution of fluorine-based organic precursors bearing the stoichiometric proportions of cations for forming RE123 is deposited on the substrate by dip-coating [23]. The technology developed at AMSC for commercial HTS wires is based on this approach. In MOD the control of the coating thickness is achieved by finetuning the solution concentration and viscosity. The coating is then dried and decomposed at temperatures below $600^{\circ} \mathrm{C}$ to remove carbon-containing materials, leaving a mixture of yttrium-barium-oxyfluoride, $\mathrm{Y}_{2} \mathrm{O}_{3}$, and copper oxides. The decomposition temperature profile is carefully controlled to prevent cracking of the film, which is $\sim 20-$ $\mu \mathrm{m}$ thick as it enters the decomposition process. This film is subsequently reacted at temperatures in the range 700$800^{\circ} \mathrm{C}$ in an $\mathrm{O}_{2}-\mathrm{H}_{2} \mathrm{O}$ environment to form RE123. A silver passivation layer, several micrometers thick, is then deposited, followed by an oxygen anneal to optimize the RE123 performance. With a single coating step it is possible to achieve $0.8-\mu \mathrm{m}$ effective RE123 thickness (corresponding to approximately $1 \mu \mathrm{m}$ actual thickness including porosity and secondary phases), but the coating and decomposition processes can be repeated multiple times to achieve thicker layers and thus increase the overall critical current. Recently, ink jet printing has appeared as an interesting alternative to the dip-coating deposition of the precursors, although only demonstrated at laboratory scale for the time being $[27,28]$. In this approach thermal or piezoelectric print heads dispense small droplets of consistent volume of the precursor solution on the substrate. This allows a better control of film thickness, precise positioning (including patterning, if necessary), solution waste minimization, and reproducibility. Presently, Oxolutia and Deutsche Nanoschicht are working to scale it up to the industrial level.

\subsubsection{Metal Organic Chemical Vapor Deposition (MOCVD)}

MOCVD is a proven process for large-scale manufacturing of several materials and it was successfully adapted to

RE123 by SuperPower [24]. It is a multistep process. Organic precursors are used for Y (or other rare earth materials), $\mathrm{Ba}$, and $\mathrm{Cu}$. In the first step, the liquid precursors are vaporized at a constant temperature. The vaporized precursors then flow in a carrier gas of argon and oxygen and are injected onto a hot substrate. RE123 films, typically $\sim 1-\mu \mathrm{m}$ thick, are deposited within a temperature regime of $700-800^{\circ} \mathrm{C}$ in partial oxygen pressure. The deposition temperature is optimized to achieve a dense film structure as well as to minimize coarsening of particulates. If the film thickness is increased, a dead layer formed by secondary phases, pores, and misaligned grains nucleates grows on the RE123 surface. However, this problem can be overcome with a multi-pass technique, i.e. depositing multiple layers of RE123 one atop each other in individual passes.

\subsubsection{Pulsed Laser Deposition (PLD)}

The PLD process, widely used in the development of the coated conductor technology, has been recently optimized by various manufacturers (Bruker, Fujikura, SuperOx and Sumitomo) and scaled up to production lengths [11, 16, 20, 21, 26]. A pulsed ultraviolet laser, typically an excimer laser from $\mathrm{KrF}$ or $\mathrm{XeCl}$, irradiated a sintered target with the desired composition to form a superconducting layer. The ablation of RE123 bulk material requires a laser fluence of $10-30 \mathrm{~mJ} / \mathrm{mm}^{2}[29,30]$ : the target material is vaporized in a plasma plume and deposited on the substrate. It is known that to insure good film stoichiometry the film thickness per one deposition pulse should not exceed 0.1 $\mathrm{nm}$. Moreover, the pulse repetition rate must be kept below $\sim 50 \mathrm{~Hz}$ to prevent deterioration of the critical current density. Bruker and Fujikura adopted a technique based on scanning the laser beam over a large-area target [18,20]. The laser plume produced in the course of such scans causes a film deposition in random sequence of small areas over a large-area substrate, which is also smoothly moved during the film deposition. The target and substrate scans 
Table 1 Fabrication process and template of industrial coated conductors

\begin{tabular}{|c|c|c|c|c|}
\hline Manufacturer & Technique & Substrate & Buffer layers & HTS \\
\hline AMSC & RABiTS/MOD & $\mathrm{NiW}$ & $\mathrm{Y}_{2} \mathrm{O}_{3} / \mathrm{YSZ} / \mathrm{CeO}_{2}$ & Y123 \\
\hline SuperPower & IBAD/MOCVD & Hastelloy & $\mathrm{Al}_{2} \mathrm{O}_{3} / \mathrm{Y}_{2} \mathrm{O}_{3} / \mathrm{MgO} / \mathrm{LaMnO}_{3}$ & Gd123 \\
\hline Bruker & ABAD/PLD & stainless steel & $\mathrm{YSZ} / \mathrm{CeO}_{2}$ & Y123 \\
\hline Fujikura & IBAD/PLD & Hastelloy & $\mathrm{Al}_{2} \mathrm{O}_{3} / \mathrm{Y}_{2} \mathrm{O}_{3} / \mathrm{MgO} / \mathrm{CeO}_{2}$ & Gd123 \\
\hline SuperOx & IBAD/PLD & Hastelloy & $\mathrm{Al}_{2} \mathrm{O}_{3} / \mathrm{Y}_{2} \mathrm{O}_{3} / \mathrm{MgO} / \mathrm{LaMnO}_{3} / \mathrm{CeO}_{2}$ & Gd123 \\
\hline Sumitomo $^{*}$ & RABiTS/PLD & $\mathrm{NiW}$ & $\mathrm{CeO}_{2} / \mathrm{YSZ} / \mathrm{CeO}_{2}$ & Gd123 \\
\hline SuNAM & IBAD/RCE & Hastelloy or Stainless Steel & $\mathrm{Al}_{2} \mathrm{O}_{3} / \mathrm{Y}_{2} \mathrm{O}_{3} / \mathrm{MgO} / \mathrm{LaMnO}_{3}$ & Gd123 \\
\hline STI $^{*}$ & IBAD/RCE & Hastelloy & $\mathrm{Y}_{2} \mathrm{O}_{3}-\mathrm{Al}_{2} \mathrm{O}_{3} / \mathrm{MgO}$ & Y123 \\
\hline
\end{tabular}

As yet marketplace conductor.

are performed independently in time, and, therefore, insure a homogeneous film thickness and stoichiometry. Under such conditions, the repetition rate of laser pulses can be increased by a factor of $10^{3}$ while the local repetition rate of the deposition pulses is kept at the low level of $<20 \mathrm{~Hz}$. The film thickness obtained by one pass is generally about 0.3-0.5 $\mu \mathrm{m}$, and thus multiple layers have to be deposited in order to acquire a high critical current.

\subsubsection{Reactive Co-Evaporation (RCE)}

In the RCE process $\mathrm{Y}$ (or $\mathrm{Gd}$ ), $\mathrm{Ba}$, and $\mathrm{Cu}$ are individually heated to evaporate and deposit them on the substrate. Atomic absorption spectroscopy is used to measure the vapor density close to the sources and fine-tune the heater power, in order to adjust the film composition. Two manufacturers adopted this approach, but with some differences. At SuNAM the film is deposited in a single step. Then, the as-deposited amorphous film is passed through a tube furnace at $860^{\circ} \mathrm{C}$ with two different oxygen pressures $\left(10^{-2} \mathrm{mTorr}\right.$ and $\left.150 \mathrm{mTorr}\right)$ and during a rapid oxidation process the amorphous film is converted in Gd123 [31, 32]. At STI the superconducting layer is formed in a cyclic process of deposition and reaction [22]. The sample alternates on a fast time scale between a low-pressure region, where it receives a deposit from the sources, and a high-pressure region ( $\mathrm{p}_{\mathrm{O}_{2}} \approx 20 \mathrm{mT}$ Torr), where it is oxidized, forming a superconducting layer. Co-evaporated materials are deposited on substrates wound on a rotating drum heated at $700^{\circ}-800^{\circ} \mathrm{C}$. As reported [22], the drum rotation is adjusted to obtain a ratio of coating time to oxidation time of 1:9. With a 5-Hz rotation speed and a 6-nm/s evaporation rate, approximately $0.12 \mathrm{~nm}$ is deposited per pass, giving an average deposition rate of $0.6 \mathrm{~nm} / \mathrm{s}$. In this way $1-\mu \mathrm{m}$ thick film can be deposited in 30 minutes.

As a closing remark to this section, it should be stressed that selecting a suitable deposition strategy for magnet applications is a very complex decision because technical aspects need to be inevitably balanced with commercial aspects and reframed within the existing market prospective. It is in fact not easy for tape manufacturers to cover the broad spectrum of specifications defined by the various industries interested in coated conductors. On one hand, the electric utility sector, representing a huge potential market for HTS conductors, has less stringent requirements in terms of single-piece length but demands both large current-carrying capacities and production capabilities. On the other hand, coil manufacturers are interested in longer, continuous lengths and enhanced mechanical properties for the market potentials much smaller than those for the electric utility sector. Achieving long, defect-free wire lengths is a true challenge that forces manufacturers to simultaneously increase the batch length and improve the process yield (defect-free length / km of processed conductor). High yield of course tremendously impacts product price especially in a small market. If production volume could be increased and the process throughput simultaneously 
enhanced, the rate at which longer lengths could be available would be also higher without necessarily working on the reduction of the number of defects per unit length. In this equation, as presented earlier in the section, throughput and batch size are the only parameters directly controllable by the wire manufacturers. Processes that allow depositing the ceramic superconductor at higher rates and over larger processing areas may, in the long run, facilitate efficient production of long lengths. A solid organic growth of the HTS conductor demands will in any case remain the main driver favouring the production of long lengths.

\subsection{Strategies to reduce the anisotropy of critical current density $\left(J_{c}\right)$}

Field-dependent $\mathbf{J}_{\mathrm{c}}$ of RE123 tape depends importantly on $\theta$, the angle between field direction and the RE123 crystallographic orientation, here, $\theta=0$ and $\theta=90^{\circ}$ defined, respectively, when field is parallel to the ab-plane (tape surface) and the c-axis (normal to tape surface). The anisotropy makes $\mathbf{J}_{\mathrm{c}}$ maximum at $\theta=0$ and minimum near $\theta=$ $90^{\circ}$ and the ratio of the current densities in the two orientations tends to increase with increasing magnetic field. By itself, the conductor anisotropy is not detrimental but it introduces an extra layer of complexity in the magnet design process. In a magnet, the conductor is exposed to a magnetic field whose angle depends on its position in the winding. This implies that the loadline at each point of an HTS coil virtually intercepts a different $\mathrm{J}_{\mathrm{c}}(\mathrm{B})$ curve. A careful adaptation of the magnet design process to the specific anisotropic conductor characteristic is necessary in order to determine criticalities and safety margins of each design.

In order to minimize the effect of anisotropy and limit the complexity of the design process, artificial pinning centers are being implemented. Over the last few years, extensive research has been conducted on $\mathrm{BaZrO}_{3}(\mathrm{BZO})$ additions. Enhanced flux pinning has been obtained by BZO-incorporated RE123 thin films made by PLD [33], MOCVD [34] and MOD [35]. Depending on deposition technique, the morphology and size of the precipitates and thus the effects on the pinning properties are different.

PLD and MOCVD lead to the formation of anisotropic nanorods ( $\sim \mathrm{nm}$ in diameter, $>100 \mathrm{~nm}$ in length) that grow along the c-axis $[36,37]$. The intrinsic weak pinning in the magnetic field parallel to the c-axis is thus reinforced by the interaction between nanorods and vortices. On the other hand, MOD produces distributed BZO-nanodots (10-50 $\mathrm{nm}$ in size), resulting in a more isotropic pinning landscape [38]. In both cases, the enhanced pinning has been correlated to the nanostrain emerging from the interfaces between the BZO inclusions and the RE123 matrix [38]. It is possible to introduce BZO inclusions also in RE123 films grown by RCE. However, the required control of the precipitates' shape and dimensions and thus the positive effects on $\mathrm{J}_{\mathrm{c}}$ are not yet achieved with this deposition technique. In a recent work on the SuNAM tapes [39] it was shown that RCE leads to the formation of uniformly dispersed $\mathrm{Gd}_{2} \mathrm{O}_{3}$ particles in the Gd123 layer. The refinement of these $\mathrm{Gd}_{2} \mathrm{O}_{3}$ particles seems to be a promising alternative to $\mathrm{BZO}$ for enhancing pinning in CCs processed by RCE [32].

In Fig. 1 the angular dependences of $\mathrm{I}_{\mathrm{c}}$ at $\mathrm{T}=77 \mathrm{~K}, \mathrm{~B}=1 \mathrm{~T}$ are compared for RE123 tapes fabricated by MOCVD with and without BZO inclusions [40]. BZO leads to a two-fold enhancement of $I_{c}$ in the orientation of B perpendicular to the tape surface (at angles around $180^{\circ}$ in Fig.1). However, at temperatures below $40 \mathrm{~K}$ BZO nanorods do not lead to any c-axis peak, even when the critical current values are higher compared with those of the undoped samples (Fig. 2) [41].

The impact of anisotropy on magnet design is very application-specific and depends from several factors like the geometry of the HTS coil itself (winding thickness, coil length, inner diameter), the presence, design and position of external magnetic sources (outserts) and the coil operating conditions (in particular temperature and ramping/cooling strategies). Specifically, for high-field magnets, the most critical regions are usually located at the extremities of the 


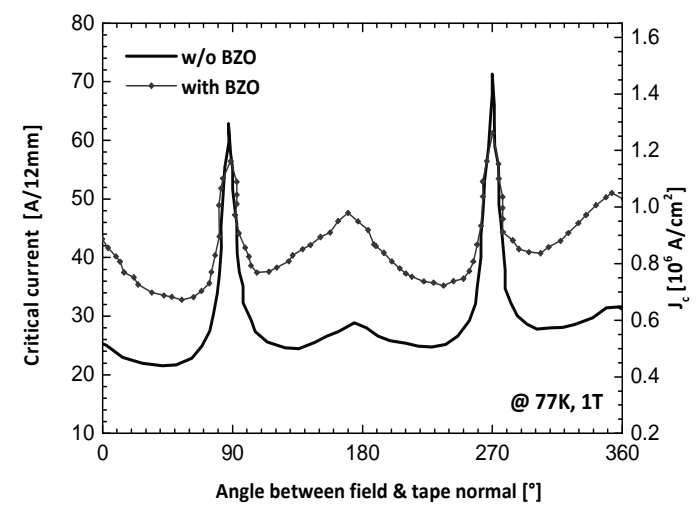

Figure 1 Effects of BZO inclusions on the angular dependence of the critical current, at $\mathrm{T}=77 \mathrm{~K}$ and $\mathrm{B}=1 \mathrm{~T}$. Data extracted from [40].

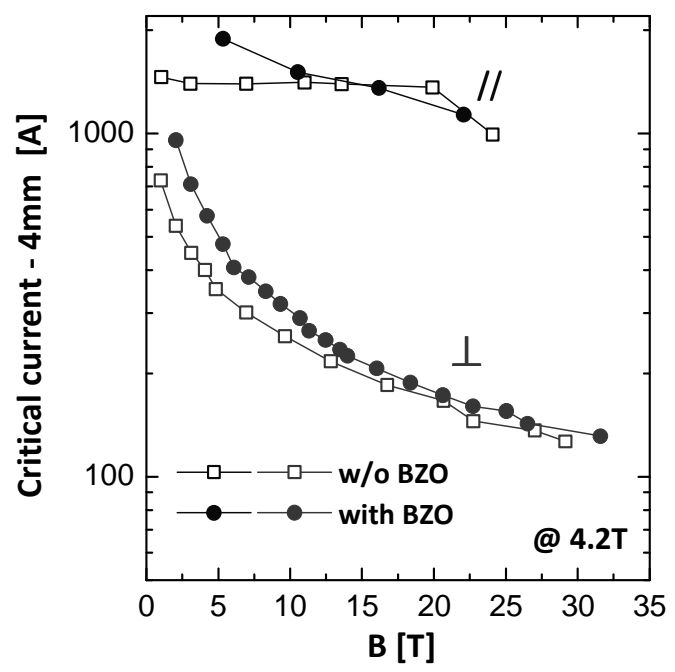

Figure 2 4.2-K Critical current $I_{c}(B)$ plots for coated conductors with and without BZO. The field is oriented parallel (blue) and perpendicularly (red) to the tape surface. Data extracted from [41].

HTS coil where the value of the field perpendicular to the crystallographic ab-plane is highest due to the combined effect of the HTS self-field and the outsert field [42].

Although not based on conductor technology, some strategies have been proposed to minimize the detrimental effects of the anisotropy. One of these, the multi-width (MW) winding technique [43], is applicable to a magnet comprising an assembly of double-pancake coils. It consists in grading the conductor width of the double pancake coils, assigning the narrowest to magnet regions near the magnet midplane and the widest to those at and near the magnet ends where, as stated above, the tape performance degradation by perpendicular field components is greatest. Generally speaking, isotropic wires are desirable mainly because not only the wire performance is independent of field direction but also the design and magnet construction processes can rely on many years of experience with LTS conductors for many magnets, e.g., high-field laboratory, NMR and MRI magnets. For this reason, the concept of round wires based on RE123 tapes to circumvent conductor anisotropy is also being examined within the European Project EUROTAPES consortium [44]: a proposed idea folds the coated conductor along its longitudinal axis into a 
tube, with the RE123 layer placed on the neutral axis of the tape. The approach is interesting but a clear drawback will be the low bendability of such a conductor.

\subsection{Strategies to reduce hysteretic losses}

For applied field above the so-called penetration field, hysteretic losses, $\mathrm{Q}_{\text {hyst }}$, in a superconducting wire scale with the critical current density and with the effective size of the filaments. The multifilamentary configuration is standard for LTS, chiefly to minimize hysteretic losses. Typical values of $Q_{\text {hyst }}$ for a field cycle $\pm 3 \mathrm{~T}$ at $4.2 \mathrm{~K}$ are $\sim 50 \mathrm{~mJ} / \mathrm{cm}^{3}$ in NbTi wires and between 100 and $1000 \mathrm{~mJ} / \mathrm{cm}^{3}$ for the various grades of $\mathrm{Nb}_{3} \mathrm{Sn}$ wires [45]. In coated conductors the superconductor is of a single layer $\sim 1-\mu \mathrm{m}$ thick and 4-12 mm width, making its hysteretic losses strongly dependent on its effective size to the direction of a time-varying field. This size varies with the conductor orientation to the field, i.e., from $\sim 1-\mu \mathrm{m}$ (parallel) to $4-12 \mathrm{~mm}$ (perpendicular). Note that in a solenoid the radial component (i.e., perpendicular direction) of its self field increases axially away from the midplane, reaching maximum at two ends of the solenoid. Therefore, at the coil ends the hysteretic losses of a coated conductor are much larger than in multifilamentary wires. The hysteretic losses depend on the effective filament size and thus in coated conductors AC losses are expected to be 2-3 order of magnitudes higher than those in LTS wires (as much as 4-12-mm wide in coated conductors against $\sim 1-\mu \mathrm{m}$ filament size in NbTi wires) and at least one order of magnitude higher losses than in Bi2212 and Bi2223 tapes, though their effective sizes are $<1 \mathrm{~mm}$, are, unlike LTS filaments, magnetically well coupled. In the perpendicular orientation $Q_{\text {hyst }}(4.2 \mathrm{~K})$ well above $10^{4} \mathrm{~mJ} / \mathrm{cm}^{3}$ have been reported for coated conductors on the IBAD Hastelloy substrate [46]. In the RABiTS approach, additional hysteretic losses arise from the ferromagnetic substrate and this is making AMSC to switch from Ni5at\%W to a non-magnetic Ni9at\%W substrate [13].

Special conductor designs are needed to minimize the conductor's susceptibility to large AC losses on perpendicular field. In particular, the subdivision of the RE123 layer into parallel filaments along the tape axis has been explored at laboratory scale either using subtractive processes, e.g., chemical etching [47], ion implantation [48] and laser ablation [49] or by direct printing of multifilamentary structures through ink jet deposition [50]. The typical width of the RE123 strips is in the order of $100 \mu \mathrm{m}$, with an inter-stripe separation of $\sim 100 \mathrm{~nm}$. In some cases striation is achieved by completely removing material, in others by degrading the superconducting properties of the inter-stripe region $[48,51]$. Also the silver cap layer and copper stabilizer on the top of the superconductor have to be striated to prevent from coupled filaments [47]. However, it is not a straightforward task to develop an economically viable process to achieve such structures over industrial lengths. Even these techniques of slitting the superconductor into many parallel narrow strips do not address to minimize another source of AC losses: coupling.

\subsection{Effects of mechanical loads on $I_{c}$}

\subsubsection{Reversible effects of strain}

Due to the intrinsic brittleness of the RE123 layer, electromagnetic strain during operation may degrade irreversibly the critical current of a coated conductor. The irreversible limits under axial and transverse mechanical loads are mainly determined by the conductor architecture and will be treated in Secs. 2.5.2 and 2.5.3. Below the irreversible strain limit beyond which the superconducting layer is damaged, strain affects $I_{c}$ reversibly, i.e., $I_{c}$ returns to its initial value when the strain is released. 

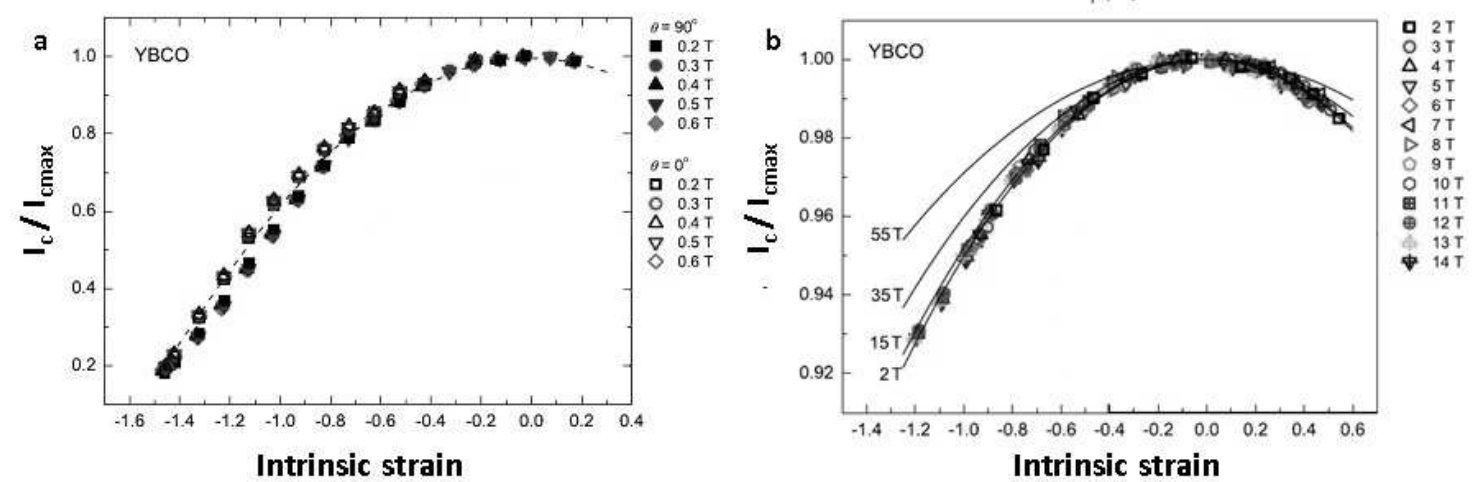

Figure 3 (a) Normalized 77-K critical current vs. intrinsic strain plots of an Y123 coated conductor for selected fields applied parallel and perpendicular to the c-axis. (b) Normalized 4.2-K critical current vs. intrinsic strain plots in selected magnetic fields in the range $2-14 \mathrm{~T}$ parallel to the c-axis. Reprinted from [52].

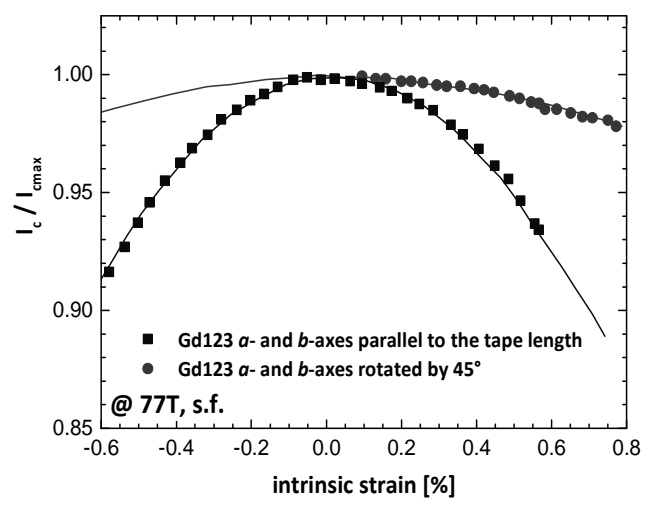

Figure 4 77-K $\mathrm{I}_{\mathrm{c}}$ vs. strain plots: blue squares correspond to a- and b-axes of the Gd123 film oriented parallel to the tape length, red circles correspond to a- and b-axes of the Gd123 film rotated by $45^{\circ}$ from the longitudinal direction [56].

As recently shown by Sunwong, et al. [52], the normalized $I_{c}$ for coated conductors follows an almost parabolic dependence as a function of strain, independent of angle and magnetic field up to $\mathrm{B} / \mathrm{B}_{\mathrm{c} 2}=0.4$ (Fig. 3). This behaviour is dissimilar to that observed in $\mathrm{Nb}_{3} \mathrm{Sn}$ wires, where the effects of applied strain on the critical current become more pronounced when $\mathrm{B} / \mathrm{B}_{\mathrm{c} 2}$ increases [53]. The difference is attributed to the stronger strain dependence of the superconducting parameters $\mathrm{B}_{\mathrm{c} 2}$ and $\mathrm{T}_{\mathrm{c}}$ in $\mathrm{Nb}_{3} \mathrm{Sn}$ compared with that in the biaxially textured RE123 layer.

The single crystal data on Y123 show that the dependence of $T_{c}$ on applied pressure is highly anisotropic within the ab-plane of the crystal lattice [54]. $\mathrm{T}_{\mathrm{c}}$ increases with pressure applied along the b-axis, $\mathrm{dT}_{\mathrm{c}} / \mathrm{dp}_{\mathrm{b}}$ being $\sim 2 \mathrm{~K} / \mathrm{GPa}$, whereas it decreases with the same rate when pressure is applied along the a-axis. In a coated conductor, RE123 grains are generally oriented either with the a-axis or with the b-axis along the conductor length and the two orientations are equally distributed. Therefore strain applied along the conductor length is locally oriented either along the a-axis or along the b-axis of the RE123 grains. This implies that the average superconducting properties of the RE123 layer do not depend on strain, but the dispersion of the distribution of the properties may change. The maximum in the $I_{c}$ vs. strain curve corresponds to an optimum strain state where the overall $T_{c}$ and $B_{c 2}$ distributions are lowest. On the other hand, critical current becomes almost insensitive to strain when coated conductors are 
Table 2 Substrate materials, electrical and mechanical stabilization of industrial coated conductors

\begin{tabular}{|c|c|c|c|c|}
\hline & AMSC & Fujikura & SuNAM & SuperPower \\
\hline SUBSTRATE & $75 \mu \mathrm{m} \mathrm{NiW}$ & $\begin{array}{c}75-100 \mu \mathrm{m} \\
\text { Hastelloy C276 }\end{array}$ & $\begin{array}{c}60 \mu \mathrm{m} \text { Hastelloy C276 or } \\
>80 \mu \mathrm{m} \text { stainless steel }\end{array}$ & $\begin{array}{c}50-100 \mu \mathrm{m} \\
\text { Hastelloy C276 }\end{array}$ \\
\hline LAMINATION & $\begin{array}{c}50 \mu \mathrm{m} \text { copper or } \\
150 \mu \mathrm{m} \text { brass or } \\
25-75 \mu \mathrm{m} \text { stainless steel }\end{array}$ & $\begin{array}{c}75-100 \mu \mathrm{m} \\
\text { one side }\end{array}$ & brass available & na \\
\hline $\begin{array}{c}\text { ELECTROPLATED } \\
\text { COPPER }\end{array}$ & na & na & $\begin{array}{c}15 \mu \mathrm{m} \\
\text { both sides }\end{array}$ & $\begin{array}{c}20-50 \mu \mathrm{m} \\
\text { both sides }\end{array}$ \\
\hline
\end{tabular}

prepared with $a$ and $b$ axes of the RE123 film rotated by $45^{\circ}$ from the longitudinal direction (Fig. 4) [55, 56]. The reversible effects of strain on $\mathrm{I}_{\mathrm{c}}$ are also influenced by other parameters:

- Strain sensitivity depends on the composition of the superconducting layer. In particular, Gd123 coated conductors exhibit a lower strain sensitivity of $I_{c}$ compared with Y123 conductors [57];

- Mismatch in thermal contraction among the different components of a coated conductor as it is cooled generates a thermal residual strain in the RE123 film, which depends on the conductor architecture;

- Peak strain value may change with applied field $[58,59]$. These changes do not depend on microscopic mechanisms related to superconductivity, but are attributed to the field and strain dependence of the normal properties of the grain boundaries [52].

\subsubsection{Irreversible limit under axial loads: influence of the conductor design}

Due to the large fractional section area of metallic substrates, mechanical strength is a big asset of RE123 coated conductor compared with BSCCO tape and wire. With stainless steel (SS) or Hastelloy substrate the critical axial stress $\left(95 \%\right.$-retention $\mathrm{I}_{\mathrm{c}}$ ) can reach, depending on the $\mathrm{Cu}$ fractional area, $700 \mathrm{MPa}$. However, the strength gap between RE123 and BSCCO conductors is reducing. It has been recently shown that the maximum tensile stress of Bi2223 tapes can be improved up to $430 \mathrm{MPa}$ by a new lamination technique [60]. On the other hand, the increase in current density of Bi2212 wires [3] leaves room for the introduction of mechanical reinforcement in the winding, without sacrificing the overall current density.

The composite architecture of the main industrial coated conductors is summarized in Table 2. Some manufacturers may apply a lamination technique to elongate the reversible strain, or just to add the required electrical stabilization. $I_{c}$ degradation of $5 \%$ is often measured at $0.3-0.5 \%$ strain, depending on the deposition technology and on the substrate. The highest strain limits may be achieved for tape laminated with a material of large thermal contraction. After the RE123 film is deposited, the substrate and the electrical stabilizer (or the laminated support), because of their large coefficients of thermal expansion (CTE), pre-compresses the film. Different precompressions are obtained by a combination of different materials of substrate, stabilizer and laminated support [61]. Interestingly, Hastelloy substrate contracts only slightly more than RE123 film, resulting in a negligible precompression induced the film.

In 2005 Shin, et al. [62], investigated the effect of stabilizer layer on critical current for a PLD-Y123 coated conductor deposited onto a 50- $\mu \mathrm{m}$ thick NiW substrate. In particular, their results showed that a 70- $\mu \mathrm{m}$ thick laminated SS tape significantly increases the irreversible tensile strain, because SS contracts more than NiW, inducing a large precompression in the superconductor layer. Apparently, the resultant precompression is large enough to be beneficial but not too large to damage the superconducting layer. The method can thus be used to 

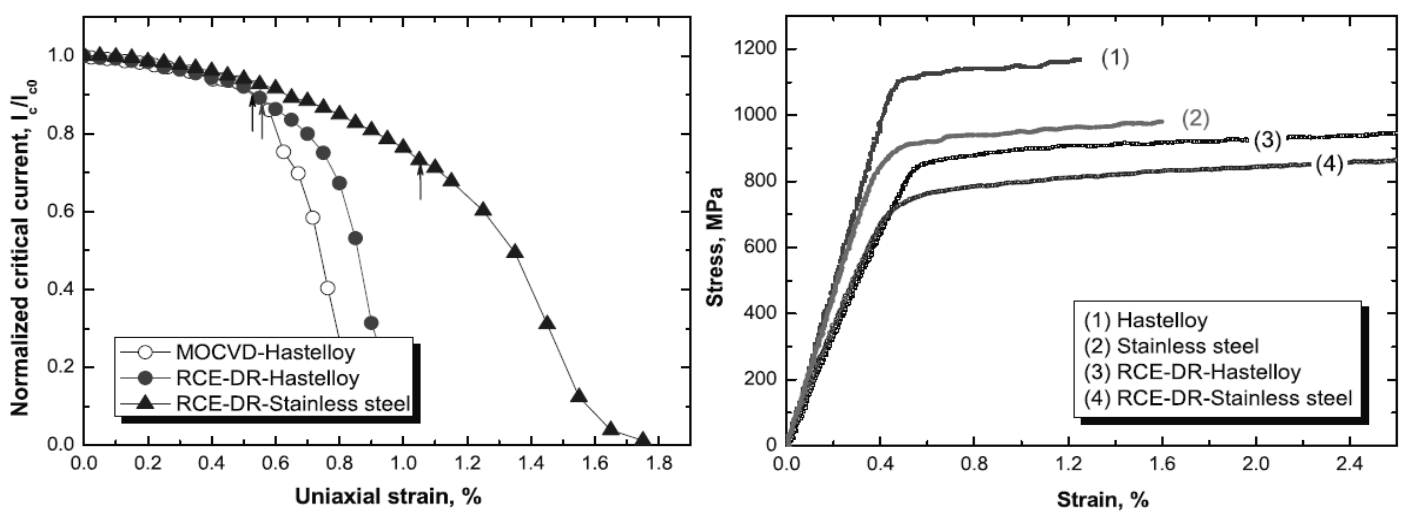

Figure 5 Normalized 77-K $I_{c}$ vs. strain curves for coated conductors with different substrates in self field (left graph). Stress-strain curves for substrate materials and Cu-stabilized coated conductor tapes. Reprinted from [65].

enhance the irreversible limit of the coated conductor. Moreover, the irreversible strain of the non-laminated tape was $0.21 \%$, while it was $0.44 \%$ for laminated tape. Sumitomo researchers have adopted a similar approach to precompress their DI-BSCCO tape by laminating the bare conductor with pretensioned tape [63].

More recently, the same authors [64] have compared the effects of a brass lamination placed on top of different commercial RE123 tapes. Results showed that brass can enhance the irreversible strain limit by more than $0.2 \%$, but clearly at the expense of engineering critical current density $\mathrm{J}_{\text {eng. }}$. In general, these data seem to evince that any lamination carried out with materials weaker than the substrate will degrade the maximum critical hoop stress at any given $\mathrm{J}_{\text {eng }}$ (referring to a solenoidal coil), even if the laminated material has a high CTE.

Regarding the electroplated copper, this stabilizer will be in annealing state as long as the temperature does not fall below $350-400^{\circ} \mathrm{C}$ after the deposition process. Only below these temperatures $\mathrm{Cu}$ will start to precompress the RE123 film. And even if the CTE of copper is very high, its influence is smaller than SS substrate (or Hastelloy), because the Young modulus of SS or Hastelloy is almost double that of copper.

Although the substrate withstands quite well high deposition temperature, the temperature dependences of its mechanical properties (Young modulus, yield stress, CTE) should be taken into account. Moreover, a SS substrate contracts more than Hastelloy (e.g. 35\% higher contraction from RT to $4 \mathrm{~K}$ ). Due to this feature, tapes with SS substrates show slightly higher strain limit for the 95\%-retention $I_{c}$ and much higher irreversible strain limits [65]. The beneficial effect of thermal contraction is also confirmed by improvement in irreversibility strain limit in tapes measured at $4.2 \mathrm{~K}$ and $77 \mathrm{~K}$ [66]. This mechanical advantage of the SS substrate is however of little importance because of the much lower yield stress of SS-Hastelloy yields at 1,100 MPa, whereas SS starts yielding at 800 MPa. Basically, even if $95 \%$-retention $\mathrm{I}_{\mathrm{c}}$ with SS substrate may fall at around $0.65 \%$ and with Hastelloy at $0.55 \%$, the corresponding tape stress would be $\sim 780 \mathrm{MPa}$ for SS and $825 \mathrm{MPa}$ for Hastelloy (Fig. 5). For very high-field solenoidal magnets, hoop stress in the superconductor is a critical parameter, and Hastelloy is likely a better support. In terms of $\mathrm{J}_{\mathrm{eng}}$ too, the thicker SS substrate becomes less preferable to the thinner Hatelloy substrate. If SS substrate is used over Hastelloy substrate, its thickness will be greater than Hastelloy's. This is to partially compensate for the lower yielding stress of SS. Generally, the larger the ratio of substrate/stabilizer, the greater the mechanical properties and the smaller the $\mathrm{J}_{\text {eng }}$.

On the other side, the SS substrate is cost-effective (it should reduce costs by $2-3$ Euro/kA m at 77K [67]), but given the today market price of the coated conductors, this advantage is now considered negligible. Another interesting point deserves to be mentioned: the deposition of RE123 on SS-316L has shown delamination problems in the past 

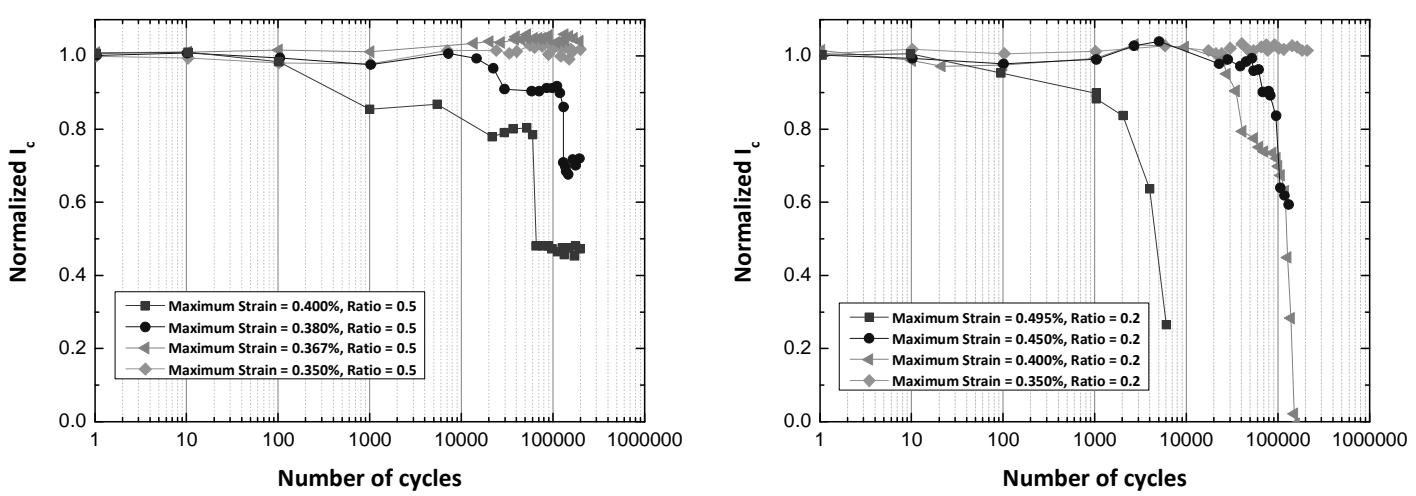

Figure $6 I_{c}(N) / I_{c}(N=0)$ versus the number of cycles $\mathrm{N}$ for samples subjected to fatigue test with a strain ratio of 0.5 (left) and 0.2 (right). Data extracted from [69].

[68] that were attributed to a strong thermal contraction mismatch between SS and RE123. From $900^{\circ} \mathrm{C}$ to room temperature SS contracts $0.4 \%$ more than RE123, while the thermal contractions of Hastelloy and RE123 are nearly the same.

Regarding enhancement of the irreversible strain limit, Cheggour, et al. [61] in 2005 reported that the stability of fracture propagation in RE123 films is strongly related to the intimate contact among the film, the substrate, and the stabilizer. It is known that the addition of metallic layers to ceramics greatly improves the fracture toughness of these brittle phases, because metallic layer acts as crack inhibiter/arrester.

Furthermore, the thicker the substrate the further away from the neutral axis of bending will the RE123 film find itself when wound on a coil former. If the RE123 film is kept on the radially-outer side (tension), it can be a problem for magnets requiring small bending radii (small radius coil formers). This is confirmed by the work discussed in [65] which indicates that a tape with $50 \mu \mathrm{m}$ Hastelloy substrate can be bent on a 6-mm radius with an $\mathrm{I}_{\mathrm{c}}$ degradation smaller than $5 \%$, whereas a tape with $100-\mu \mathrm{m}$ SS substrate already degrades by almost $10 \%$ when bent on a 6 -mm radius, thus showing a much higher sensitivity to bending strain because of the higher intrinsic strain applied to the RE123 film.

Among available literature a few research efforts has covered also the effect of cyclic loads. Superconductors used in magnet applications are exposed to various mechanical loads during their operational lifetime. These loads are mostly due to handling during manufacturing, thermal contraction mismatches during cooling and electromagnetic forces during operation. Features such as intensity and frequency of the loads strongly depend on magnet application. Laboratory and NMR magnets are usually ramped up slowly with load frequencies of orders of magnitude lower than those found in electrical machines (motors and generators). Nevertheless, design of durable high field laboratory magnets must take into account the effect of cyclic loads on the critical current. Fatigue occurs when a material is subjected to repeated loading and unloading. Coated conductors are manufactured with both ductile metals and brittle ceramics, and their interaction under repeated loads is cumbersome. The superconducting ceramic is extremely sensitive to the maximum applied stress, and much less to the fatigue stress ratio, i.e. the ratio between minimum and maximum stress experienced in a cycle, whereas the ductile metals could easily present fatigue strength limit in the order of $1 / 3$ of the static one just for being exposed to large fatigue stress ratios. High stresses have higher probabilities to quickly damage the ceramic compound, whereas large fatigue stress ratio can slowly generate cracks in the metals of the composite tape. Mbaruku et al. [69] were the first to characterize commercial coated conductors in cycles $>100 \mathrm{k}$. In particular, their work has pointed out that fatigue cracks in the RE123 layer do not occur 
everywhere in the sample but are localized in weak areas, starting from the sample edge and moving towards the center (likely due to the slitting process used to cut a $12 \mathrm{~mm}$ wide tape into 3 strips of $4 \mathrm{~mm}$ width). Their results on a standard SuperPower tape with 50- $\mu \mathrm{m}$ thick Hastelloy substrate and $20-\mu \mathrm{m}$ thick Cu stabilizer show no degradation in $I_{c}$ or n-values even after $200 \mathrm{k}$ cycles for an applied maximum strain of $0.367 \%$, with a fatigue strain ratio of 0.5 at a load frequency of $0.4 \mathrm{~Hz}$. Significant degradation was observed with higher maximum applied strains, or with smaller strain ratios (see Fig. 6). Sugano et al. [70] reported results on samples fabricated by MOCVD on a 100- $\mu \mathrm{m}$ thick Hastelloy substrate. They found two types of fatigue mechanisms acting independently from each other: one related to failure of the Hastelloy substrate and the other related to failure of the Ag layer. More recently, Shin et al. [71] tested IBAD/ Sm123 tapes deposited on a 80- $\mu$ m thick Hastelloy substrate. Their tests have confirmed once again that the fatigue cycle limit decreases drastically at smaller strain ratios. All these tests indicate that not only a long lifetime can be expected for standard laboratory magnets but also a maximum strain should be chosen carefully for magnets that will undergo many loading cycles.

\subsubsection{Transversal stress and delamination}

The delamination problem of coated conductors has been known for a few years now. Indeed, RE123 coated conductors are made up of numbers of layers which can be modelled as adhesive lap joints, where the brittle buffer/RE123 layer is expected to bond both the substrate (on which it is deposited) and the silver layer, on top of which the copper stabilizer is usually deposited or laminated. The RE123 coated conductor performances are degraded significantly once the brittle adhesive buffer/RE123 assembly layer detaches or breaks. Five common stresses inherent in adhesive joints can cause detachments/fractures: tensile, shear, compressive, cleavage, and peel. Tensile stress corresponds to hoop stress in a solenoidal coil. Cleavage occurs when an external force acts to open one edge of the adhesive assembly. Peel is similar to cleavage, except here an adhered part is flexible. In cleavage and peel, the detaching force acts over a small part of the adhesive bond and, therefore, the critical (cleavage and peel) strengths are much lower [72]. Recently, Maeda at Riken summarized all the mechanical properties of RE123 tape with a detailed matrix reported in Table 3 [73]. The axial tension of $>700 \mathrm{MPa}$ refers to a 50- $\mu \mathrm{m}$ thick Hastelloy substrate tape. Also note that each stress value corresponds to a tape with electroplated copper stabilizer.

The initial work by NIST in 2007 [74] on tapes produced by AMSC suggested that the tape slitting procedure in the production process plays an important role in the delamination issue. In order to reduce the manufacturing costs of coated conductors, many manufacturers deposit a thin film on substrates a few-cm wide. This wide tape is then slit longitudinally to produce standard tapes, e.g. 4-mm wide. This slitting deforms the substrate, which is clearly visible in micrographs of the slit tape cross section, and also generates initial cracks in the RE123 layer that can easily propagate under a relatively small applied transverse tension. A transverse tension can in general occur on the conductor if the winding is filled with, for example, epoxy resin with unmatched thermal contractions. Without precautions, epoxy impregnation, which is a common practice in magnet technology (especially in the power sector), can easily damage the coated conductors. In the same paper, NIST suggested that the strength of slit coated conductors against delamination raised significantly by reinforcing the conductor with laminated copper strips and solder fillets at the conductor edges. They also noted that islands of relative weak material, invariably present in the conductors, result in strong $I_{c}$ degradation at low transverse stress. These local microstructure weak spots may originate from production defects or during slitting procedure or both. Finally, the NIST authors argued that coated conductors lack a soft metallic layer that could act as an absorber to compensate for thermal mismatch during cooldown (epoxy resins contract much more than metals); on the other hand, Bi2223 tapes have a matrix of silver, a 
Table 3 Stress limits for a coated conductor under various mechanical constraints [73]

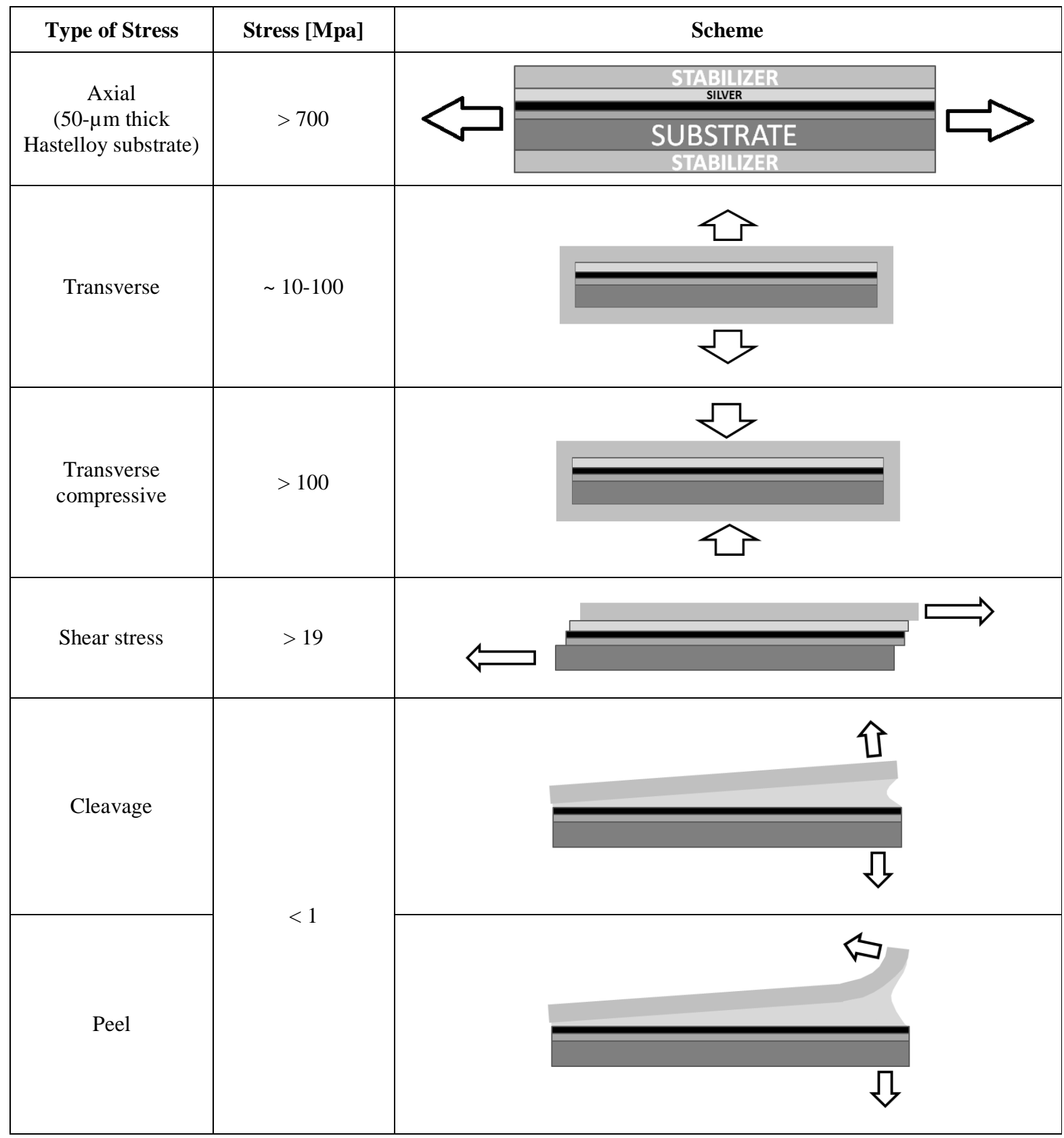

metal much more malleable than SS or Hastelloy. Indeed, delamination has never been an issue with Bi2223 coils (even if delamination tests showed strengths lower than those in coated conductors). The silver deforms without transferring the thermal stress to the brittle ceramic filaments of $\mathrm{Bi} 2223$.

In order to circumvent the delamination problems in epoxy impregnated layer wound coils, NHMFL proposed to use Polyester heat-shrink tubing from Advanced Polymers, Inc. [75] to decouple the tape from the epoxy. A similar problem was observed in epoxy-impregnated double pancake coils by Takematsu, et al. [76]. A thorough investigation on the subject, including tests and FEM analysis, was carried out by Yanagisawa, et al. [72]. This research group [77] found that degradation of the epoxy impregnated RE123 coil is eliminated if a polyimideelectrodeposited (PIED) insulation of thickness $15-30 \mu \mathrm{m}$ is applied to the tape conductor. The transverse stress on 
the tape is reduced owning to 1) plastic deformation of the ductile polyimide and 2) easily debondable epoxy resin. The polyimide layer acts as a "missing" soft absorber layer that apparently accommodates thermal mismatches during cool-down. To date, this solution seems to be the best option for insulated coils [78, 79].

Other investigations at $\mathrm{UH}$ and Andong University [80, 81] indicate that if the problem must be fixed at the manufacturing stage, a viable solution would be to make the electroplated copper layer thicker, especially along the tape edges, which the slitting may have damaged.

For high-field magnet applications, our focus should be on developing suitable insulation and/or impregnation techniques rather than eliminating defects during the deposition process. All the literature available suggests that in case of epoxy-impregnated coils, it is safer to decouple conductor and epoxy; this decoupling may be unnecessary with PIED insulated tape. The technological consequences of delamination and impregnation are further discussed in section 3.1.3, "Coil impregnation".

\section{Engineering issues in 2G HTS coil technology}

\subsection{Coil Technology}

For the HTS magnet to fully achieve its potentials, the intrinsic anisotropic properties of RE123 and large aspect ratio of its tapes pose design challenges, requiring solutions, both innovative and based on the traditional LTS technology. The relevant technical challenges posed by the HTS conductors for magnet applications are winding process, coil impregnation, insulation, and splicing.

\subsubsection{Winding Techniques}

Two widely used techniques to wind solenoids are layer-wound (LW) and pancake (P)/double-pancake (DP). In an LW coil, the conductor is wound turn by turn one layer at a time, from the innermost to outermost layers [82]. Generally, the conductor should be long enough to complete at least one full layer. The LW allows the entire coil to be wound with a single conductor piece, provided the conductor is long enough. In a DP coil, applicable to a highaspect-ratio conductor, e.g., tape, two pancakes are wound sequentially, each pancake starting from the conductor midpoint and spiraling radially outward from the innermost to outermost turns [83]. The "cross-over" form one pancake to the other at their innermost turns is continuous, i.e., no splice. Because the winding height of each DP coil is about twice the conductor height ( $\mathrm{h} \geq \mathrm{w}$, where $\mathrm{w}$ is the tape width), a "long" magnet is an assembly of DP coils. The adjacent DP coils are spliced in the radial space outside the outermost winding diameter. Generally, a DP coil requires a continuous length of conductor that is considerably shorter than that required in a typical LW coil, e.g., even a $50 \mathrm{~m}$ long conductor may suffice for a DP coil. Even in a very large magnet, a continuous length of $<1 \mathrm{~km}$ should be sufficient for a DP coil in contrast to that exceeding $10 \mathrm{~km}$ required for an LW magnet. At present, HTS tape longer than $200 \mathrm{~m}$ remains a challenging production hurdle, making DP technique a valuable option for HTS magnet. In this early stage of HTS magnet technology, modularity and scalability, both intrinsic to the DP technique, are attractive features to avoid risks of catastrophic mistakes that may occur during winding and testing. Both LW and DP techniques are being used to wind HTS high-field magnets (see Section 4).

\subsubsection{Critical aspects of winding}

As extensively discussed in Sec. 2.5, a combination of a thin ceramic biaxially textured RE123 layer and a mechanically tough substrate (Hastelloy, NiW or stainless steel) makes the $2 \mathrm{G}$ HTS tape mechanically viable to the 
high-field magnet, in particular an extremely high axial tensile stress of up to about $700 \mathrm{MPa}$ [84]. Nevertheless, due to a large anisotropic stress tolerance, HTS tapes can be delaminated and degraded by a transversally tensile stress of only 10-20 $\mathrm{MPa}$ [72]. In coated conductors, independent of the fabrication process (RABiTS, IBAD), the superconducting layer is fully formed and present when a coil undergoes the react-and-wind process. In order to preserve the tape's superconducting performances, the conductor strain must be kept below a critical limit. The importance strains are: bending during the winding process $[85,86]$; cleavage by differential thermal contractions, particularly in an epoxy-impregnated coil [72]; and electromagnetic hoop stress (see Sec. 3.1.6).

When a coil is wound, the bending strains occur along two principal directions: a) conductor axis ("easy bending"); b) normal to the conductor axis ("difficult bending"), at the inner transition region in a double-pancake coil or each end of an LW coil. From the bending analysis of thin beams, Markiewicz, et al. modelled the winding strains of DP and LW coils, derived analytical expressions for the easy and difficult bending strains, and compared their results with the measured irreversible strains [87]. For large winding diameters, bending strains are generally low but become a design constraint for those smaller than $50 \mathrm{~mm}$. Joints are typically located in low-field regions. Note that, unless a great care is exercised, a great bending strain can occur in a joint [87].

\subsubsection{Coil Impregnation}

In an LTS solenoid magnet, impregnation (e.g., epoxy, wax [82]) transforms a void-filled winding into a solid monolithic structure (mechanical load is uniform only in the axial direction), eradicating or minimizing conductor motion, a chief source of premature quenches in nearly all "adiabatic" LTS magnets in the 1970s and early 1980s. In coils wound with coated conductors, because of the very large intrinsic temperature margin, the energy released by tape motion is unlikely to induce quenches. Impregnation immobilizes also the conductor against electromagnetic forces - immobilization may prevent stress concentration that results when the conductor moves — and may enhance effective conductive thermal conductivity within the winding, an important benefit especially for coils with a temperature gradient, such as conduction-cooled coils. Nevertheless, at present, most high-field HTS coils are still designed for operation in a bath of liquid He.

The mismatch of the thermal contraction coefficients among former, epoxy and HTS conductors generates thermal stresses that occur mainly around the edge of the conductor [72]. Already in a relatively small pancake coil [76, 88] the thermal stress is enough to induce delamination of the superconducting layer. In the long term the manufacturers of coated conductors may succeed in developing tapes with improved bonding between the layers, but alternative techniques dealing with the impregnation process of the commercial tapes presently available have already been reported. For example, in order to avoid the thermal stress at the edge of the conductor, Hwanjun Jeong, et al. [89] suggested reducing the width of the RE123 layer of $30 \%$ with respect to the tape width, but this is applicable only to tape which are not slitted after deposition. In general delamination may best be avoided by reducing the adhesion between impregnating material and tape. For the epoxy-impregnated winding, it has been shown that insulating the coated conductor with polyester heat-shrink tubes [75] or electrodeposited polyimide [77] can solve the problem. Another possibility is to modify the coil design: if the magnet is of a nested-coil formation, with each coil's radial build sufficiently thin, the thermal stress is strongly reduced [90].

Another option is to replace epoxy resin with materials of weak bonding strengths. In principle any liquid material that solidifies after the application could be used for filling up voids and obtaining a monolithic structure. For example paraffin and/or bee wax have been used to impregnate coated conductor coil without any major problem [91]. Although several research groups use epoxy impregnation for high-field solenoid coils, epoxy impregnation is 
not compulsory. Indeed most MRI and NMR magnets are impregnated either with wax or epoxy resin. Another option is cyanoacrylate resin [92], which has also proved to have a sufficiently low adhesion and used successfully for impregnating racetrack coils [92]. One more possibility is to choose a former material of an extremely low thermal contraction that will reduce thermal stress [93].

KIT proposed [94] a blend of araldite epoxy with quartz powder in a 1:1 ratio for degradation-free impregnated RE123 tapes/coils, targeted to Roebel cables. The blend is not the best match with RE123 conductors in terms of thermal contraction from RT to $77 \mathrm{~K}$ nor has its effectiveness against delamination been demonstrated with full-scale coils. Furthermore, because the filler generally makes the epoxy denser and more viscous, the epoxy-impregnated DP coils of $\geq 4$-mm wide superconducting tape are unlikely to be $100 \%$ void-free unless the wet winding technique is used.

Finally, it should be pointed out that a coil may also be constructed impregnation-free, i.e., dry winding. Winding tension makes the coil structure a more solid. This is the approach adopted by the NHMFL to construct a $32 \mathrm{~T}$ magnet (see Section 4).

\subsubsection{Coil Insulation}

Finding suitable insulation for coils wound with a large aspect ratio tape such as the coated conductor is very challenging. It must operate under severe conditions typical of a superconducting magnet: cryogenic temperatures; large electromagnetic stresses; high voltages developed during quenching. Different techniques have been adopted in prototype HTS magnets depending on their tape, geometry and manufactory method. The Bi2223 manufacturer has proposed Kapton, the "traditional" insulator for Bi2223 tapes: a thin Kapton tape with or without adhesive is wrapped around the Bi2223 tape, with an overlap that could be specified by the customer. The coated conductor manufacturer has also proposed Kapton. Kapton, weak mechanically, has one additional disadvantage: its thickness is comparable to the coated conductor's. It thus nearly halves the overall current density of the DP coil. As the outer layer of coated conductors is in general of copper, Kapton may be replaced with enamel [95], a widely used insulation for the NbTi wire. To avoid degrading the coated superconductor, a caveat in applying enamel to the superconductor is to keep its curing temperature below $300^{\circ} \mathrm{C}$ and limit the duration to a few minutes. At IRL [96] a UV curable varnish insulates slitted strands used for Roebel cable. At NHMFL different insulation techniques have been tested, among them thermally-cured and UV-cured polymer coatings, oxide physical vapour deposition, and (on co-wound steel tape) oxide sol-gel coating: a production system of UV-cured epoxy coating and an experimental solgel coating system have been successfully set up [97]. Other interesting approaches reported are the polyester heatshrink tubing of Trociewitz, el al. [75] and the electro-deposited polyimide insulation of Yanagisawa [77], both described in Sec. 2.5.3.

In double-pancake coils the tape may be insulated only on one side (layer-to-layer insulation by mean of co-winding an insulating tape), with solid spacers providing the insulation between pancakes. At NHMFL each double pancake of the 32-T magnet is co-wound, sans impregnation, with sol-gel alumina-coated stainless steel tape. Considering a low radial thermal conductivity of the pancakes, Bai, et al. [98] suggested that copper cooling disks may be required to promote radial heat flow. BNL also investigated co-winding with bare SS tape and concluded that the low electrical conductivity of steel is sufficient to provide turn-to-turn electrical insulation [99, 100]. The pancakes are dry wound but the top and bottom sides are coated with epoxy before, which is an interesting solution to provide mechanical reinforcement and it may be usueful for quench protection. Weijers, et al. isolated the DP by co-winding with insulated steel, and subsequently vacuum- impregnating with unfilled Stycast 1266 epoxy [97]. A 25-50- $\mu \mathrm{m}$ 


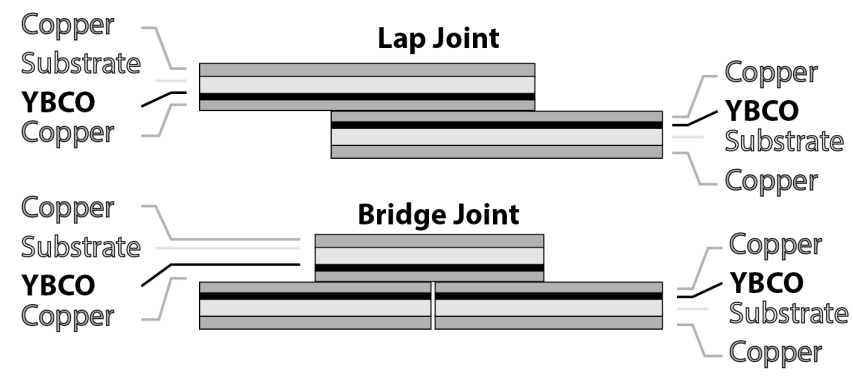

Figure 7 Different joint configurations: Lap joint (top) and Bridge Joint (bottom).

thick Kapton layer separated the pancake halves. When the pancakes were stacked and mounted on the bore tube, the selected units were coated with Stycast 2850 at the i.d. and top/bottom surfaces. Yu, et al. used 0.05-mm thick, 12.7mm wide Kapton for turn-to-turn insulation for 0.1-mm thick, 12-mm wide RE123 tape. Each turn of the coil has one layer of the Kapton co-wound at the bottom of the superconductor, thus the fraction of the cross section occupied by the insulation is about $34 \%$ [101].

The coated conductor manufacture is currently developing a substitute for Kapton, and the conductor with alternative insulation may become available within the next few years.

The production of no-insulated coils (see Section 3.2.1) is being investigated extensively in the last few years. It might be a viable option, if the presence of interlayer electrical resistances is not detrimental to operation. Important operational issues for no-insulated coils include magnet charge sequence, quench protection, and fault-mode unbalanced forces.

\subsubsection{Splicing}

Because not only the commercially available coated conductor is currently limited to lengths less than $\sim 300 \mathrm{~m}$ but also a magnet comprising an assembly of double-pancake coils requiring many joints, a splicing technique, lowresistance, easy-to-make, and reliable, is required. In LTS materials ( $\mathrm{NbTi}$ and $\mathrm{Nb}_{3} \mathrm{Sn}$ wires) techniques to make superconducting joints are now widely available. The ceramic nature of coated conductors and their extreme sensitivity to oxygen and high temperatures preclude typical metallurgical jointing approaches, proven successful with LTS. To date, a technique developed recently by Y.J. Park, et al. [102] gives encouraging results. This approach consists in sintering two counter-facing ceramic layers in vacuum $\left(>800^{\circ} \mathrm{C}\right)$ and restoring the optimal oxygen content by a special heat-treatment performed at a lower temperature. The high-temperature process is short but the annealing process to re-inject oxygen in the conductor is quite long, over 200 hours. Haigun Lee has recently shown that the re-oxygenation of Y123 during annealing can be facilitated by opening micro-holes on the surface of the tapes with a laser beam. This approach has been successful in restoring the full $\mathrm{I}_{\mathrm{c}}$ of the tape after the jointing process: A current of $\sim 60 \mathrm{~A}$ induced at $77 \mathrm{~K}$ in a loop containing a joint remained at the same current after more than 200 days [103]. For a magnet comprising double pancake coils, making each joint between adjacent double pancake coils superconducting is a must for persistent-mode operation. Non-superconducting joints can have more flexible geometries, including the lap joint, where two end faces are butt-joined, and the bridge joint, where two HTS tapes are bridged (see Fig. 7). The electrical resistance of a soldered joint depends on such factors as HTS tape lamination (brass or copper), solder material and layer thickness, surface cleanliness, contact uniformity, and pressure at the time of soldering. The solder resistivity, because solder is an alloy, does not decrease more than a factor of 2 from 77 

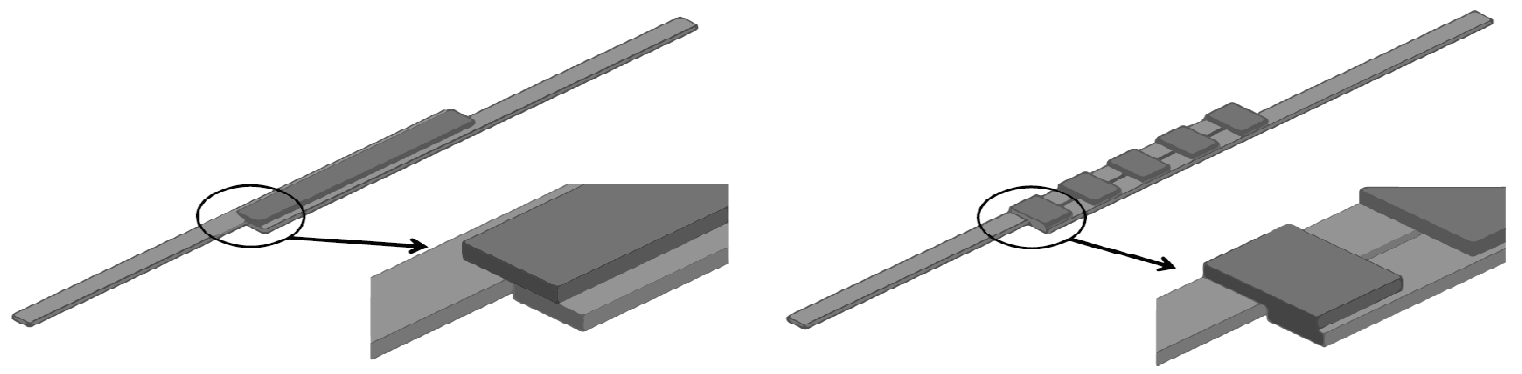

Figure 8 Different configuration of bridge joints. Left: PD joint; right: OD joint.

$\mathrm{K}$ and $4.2 \mathrm{~K}$. Another important point with the coated conductor is that the "superconductor side" surface rather than the "substrate side" surface must be soldered.

Splicing method, solder material and flux, temperature must match the specific characteristic of the tape: in laminated tape the temperature should be sufficiently low to avoid dismembering the laminated tapes (AMSC and Fujikura RE123; Sumitomo Bi2223). Furthermore, RE123 is deteriorated, by losing oxygen, when heated for a period of time, e.g., $>1 \mathrm{~min}$ at $300^{\circ} \mathrm{C}$. These inherent dangers are reflected in the soldering recommendations of major manufacturers such as AMSC and SuperPower [104].

Among many reports on preparation of resistive joints, one of the most complete is Duckworth, et al. [105]. Various solders and fluxes were tested for RE123 joints in several configurations. The best results are achieved with twocomponent alloys, e.g., $\mathrm{SnAg}$, InSn, $\mathrm{SnPb}$, with RE123 pre-tinned or with an aluminium heater block to press the joint surfaces during the soldering process. The specific resistivity, defined as the resistance times the joint area, is a useful parameter. In general, measured specific resistivity at $77 \mathrm{~K}$ ranges $<30 \mathrm{n} \Omega \mathrm{cm}^{2}$ to $>500 \mathrm{n} \Omega \mathrm{cm}^{2}[106]$.

Ko, et al. report results of lap joint and bridge joint that connects two parallel conductors [107]. Of the bridge joint, there are two types, parallel direction (PD joint) and orthogonal direction (OD joint), depending on the direction of the "bridge" tape with respect to the direction of the parallel CC tapes. The HTS layers of CC tapes faced each other with $75 \%$ of each CC tape covered with the bridge tape, as illustrated in figure 8. With a soldering machine developed by themselves [108], they made each joint at $190^{\circ} \mathrm{C}$, pressing it at a pressure of $17 \mathrm{~kg} / \mathrm{mm}^{2}$, and cooled it down to room temperature in 15 minutes [95]. Their bridge joint $\left(240 \mathrm{~mm}^{2}\right)$ contact resistivity and critical current range, respectively, $96 \mathrm{n} \Omega \mathrm{cm}^{2}-336 \mathrm{n} \Omega \mathrm{cm}^{2}$ and 90 A-100 A. Although in general, PD joints were better than OD joints, in terms of performance (contact resistivity and critical current) either type fares less well compared with the lap joint.

In Section 2.5, the effect of mechanical loads on bare tape performance was discussed in detail. For practical applications splices are absolute necessity. The splices are also subjected to electromagnetic forces. The force on splices has been investigated with techniques similar to those used to characterize single bare tapes [109, 110]. These studies have shown that the splice's tensile strength may be strongly influenced by the tape architecture. In fact soldered splices without copper stabilizer do not carry full critical currents [109]. Encouragingly, splices of copper-stabilized tapes carry full critical currents up to about $0.5 \%$ strain [110]. The source of failed splices is generally the same as that identified in bare tapes: at the interface between buffer layer and RE123. For this reason, strategies that reduce stress peaks at the splice and vicinity may enhance the mechanical properties of the splice.

Independently from the tape architecture (i.e. with or without copper stabilization) it has been demonstrated that the soldered splice resistance is robust and independent of tensile stress. 


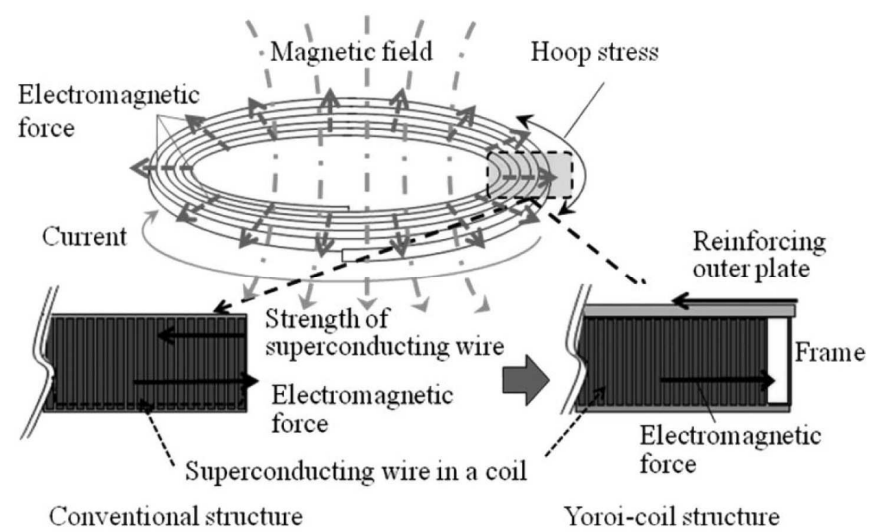

Figure 9 Schematic of supporting mechanism of "Yoroi-coil" structure against electromagnetic force. Reprinted with permission from Nagaya et al. Applied Superconductivity, IEEE Transactions on 234601204 [113], Copyright 2013, IEEE.

\subsubsection{Stress Management}

The use of coated conductors in high-field superconducting magnets requires high strength against electromagnetic forces. In solenoidal magnets the hoop stress is tensile along the longitudinal direction of the conductor. Since the current carrying capabilities of these ceramic films are strain sensitive, it is necessary to limit the conductor strain. The conductor substrate metal should be strong enough (a high elastic modulus and a large elastic strain limit) to bear most of the hoop stress to limit the strain on the superconducting film. From a conductor point of view, the reinforcement options are described in Section 2.5. Examples of peak stresses in high field magnets are reported in Section 4.

If the hoop stress is to exceed the substrate strength (or more in general the composite conductor strength, as the tensile stress can also be partially supported by the stabilizer or by the metal tape laminated on the conductor), then an additional reinforcement element must be provided.

Co-winding a high-strength strip is an oft-used reinforcement technique. The added material reduces the winding pack overall current density, but allows the conductor to safely carry full operating current. Each design, however, is a comprise among competing requirements. An example is the double pancake design for the $32 \mathrm{~T}$ project at NHMFL [111], where an insulated steel tape is co-wound with the superconductor. Alternatively, the overwrap can be entirely wound over individual double-pancake coils, an example of which is a 100-T all-superconducting magnet [112].

For magnets designs not requiring high spatial field homogeneity, other stress management designs are possible. For an SMES unit, Nagaya, et al. [113] are developing the "Yoroi-coil" structure, where a part of the electromagnetic force is shifted from the conductor to the reinforcing outer plates situated at the side of the coil (Fig. 9). Using the Yoroi-coil structure concept, they demonstrated that it would be possible to push a calculated magnetic hoop stress of a $100-\mu \mathrm{m}$ thick Hastelloy-backed conductor to beyond 1.6 GPa vs. a typical conductor limit of 1.3 GPa [114]. The measured conductor strain of this test coil was just $0.4 \%$, suggesting that it would be possible to reach magnetic hoop stresses above $2 \mathrm{GPa}$ without any irreversible degradation of the RE123 film. This concept seems to be better suited to pancake magnets, but it should be possible to apply this concept to other coil configurations.

As a final remark, given the inconsistency between tests conducted on straight samples and tests conducted with applied electromagnetic forces on 1-turn coils [114], it is possible to argue that the peak hoop stress limit for coated 
conductor has yet been precisely established and therefore it is recommended that the magnet designer be careful in pushing these conductors close to their mechanical limits.

\subsection{Quench Protection}

As in most devices, the superconducting magnet must be protected against fault mode that may lead to permanent damage. One common fault mode, particularly among LTS magnets, is a quench. The first step in quench protection is the detection of a quench, the most widespread detection technique being the resistive voltage that results from the quench. For a driven-mode magnet, the protection phase consists of two events: first switching off the power supply; and second automatically dumping most of the stored magnet energy into an external "dump" resistor, thereby to keep the initial hot spot from being overheated. The various protection options are discussed in [82]. In the so-called self-protecting magnet the stored energy is dissipated within the magnet winding without external intervention. In case of passive protection, common among persistent-mode, adiabatic LTS magnets, the protection system is within the cryostat: typically diodes and shunt resistors are paralleled across the nested coils comprising a magnet. Examples include laboratory as well as NMR and MRI magnets. In another active protection system, quench heaters placed within the winding are activated to intentionally quench a large enough winding volume, again, to keep the hot spot from being overheated.

One issue in protection of adiabatic coils is the normal zone propagation velocity (NZPV), which in the HTS winding is much slower, by one to two orders of magnitude, than in the LTS winding [115-117]. This is because at a given operating current and magnetic field the NZPV is inversely proportional to the square root of the temperature margin, which is clearly much larger than in classical LTS materials. Adiabatic LTS coils are usually operated very close to the critical surface, i.e. their smallest temperature margins within the winding are often a fraction of a kelvin. When a normal zone appears in the adiabatic LTS winding it will spread very quickly along a large section of the coil. In the HTS winding, because of its low NZPV, the voltage across a normal zone will be low, making quench detection challenging in general and protection in particular [82].

The NZPV and minimum quench energy (MQE) have been measured for coated conductors test coils in the temperature range 30-80 K (mainly for power applications), but the data are of limited interest for high-field magnets operating at liquid helium temperatures. Measurements on short coated conductor samples at $4.2 \mathrm{~K}$ were carried out and the results compared with those for $\mathrm{Bi} 2212$ and $\mathrm{MgB}_{2}$ conductors [118]: at the same reduced current $\left(\mathrm{I} / \mathrm{I}_{\mathrm{c}}\right)$ the NZPV and MQE of coated conductors and Bi2212 wires are comparable (for example about $40 \mathrm{~mm} / \mathrm{s}$ at $\mathrm{I} / \mathrm{I}_{\mathrm{c}}=0.6$ and about $100 \mathrm{~mm} / \mathrm{s}$ at $\mathrm{I} / \mathrm{I}_{\mathrm{c}}=0.8$ ), while for $\mathrm{MgB}_{2}$, which has a critical temperature of $39 \mathrm{~K}$, considerably lower than those of RE123 and Bi2212, the respective values are 20 times faster and 5 to 20 times lower.

A very interesting approach to speed up the NZPV in coated conductors is based on increased contact resistance between the superconducting layer and the stabiliser/substrate [119]. Indeed the interface next to the superconducting layer appears to be a key player in NZP [120]. The numerical simulation of Badel, et al. [119] shows a drastic increase of NZPV when the thermal conductance of the superconductor/substrate interface becomes lower than 40 $\mathrm{W} / \mathrm{K}-\mathrm{cm}$, i.e. when the superconducting layer is almost thermally decoupled from the substrate. Similarly Levin, et al. proposed [121], on the basis of numerical simulations, that increasing superconducting-layer/stabiliser contact resistance would increase NZPV. Following these indications the modification of an industrial tape from Superpower was carried out by Lacroix et al. [122]: the superconductor layer is separated from the $\mathrm{Ag}$ and $\mathrm{Cu}$ layers with a proprietary layer, deposited before the metal matrix layers. The dramatic effect of this layer may be seen from a graph of 77-K NZPV vs. current plots on selected contact resistances, shown in Fig. 10. One drawback is an added, 


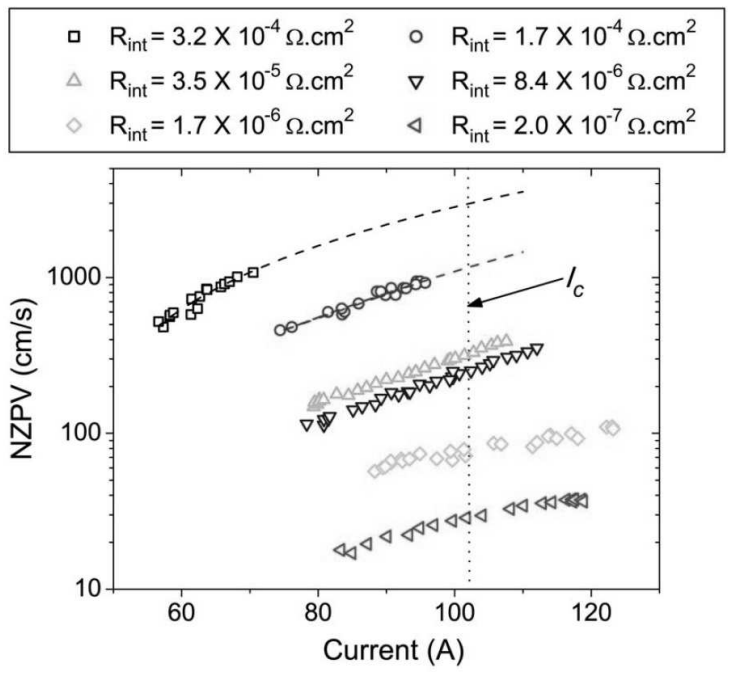

Figure 10 Dependence of NZPV with applied current for different values of the interfacial resistance between the ceramic layer and the stabiliser. Reprinted with permission from Lacroix et al. Applied Superconductivity, IEEE Transactions on 234701605 [122], Copyright 2013, IEEE.

and perhaps more complicated, manufacturing step. Another is reduced stability. Still, it may be worth paying these prices of reduced stability, though the efficacy of this higher contact resistance should further be proved at LHe temperatures in coils rather than only on short samples. In another effort to speed up NZPV, which is a complete opposite of the above, the thermal conductivity within the coil pack was improved with a coat of $\mathrm{ZnO}$ [123]. The results were negative with epoxy-impregnated coils, possibly because epoxy might have degraded the overall thermal conductivity. In a more recent experiment [124] titania-doped electrical insulation led to a 2.5 factor increase in NZPV.

With respect to quench detection, optical fibres are used to detect a quench-induced temperature rise [125]. An electromagnetic method involving a special preparation of the tape [126] and acoustic emissions (AE) [127-132] have also been investigated. Except AE technique, which has proven valuable and applied to diagnose the quench sources in adiabatic LTS magnets, the other approaches remain in R\&D stage.

One important aspect that should be mentioned is that in the design of LTS magnets the amount of stabilizer (in general copper) is an important parameter, which is optimised for each magnet. In contrast with this, the copper cross section in the commercial coated conductor is much smaller than those in LTS wires. For example, in the standard 4mm wide SuperPower coated conductor with $20-\mu \mathrm{m}$ thick plated copper layers, the total copper cross section is 0.16 $\mathrm{mm}^{2}$; since the tape can carry more than $200 \mathrm{~A}$ in a high field at $4.2 \mathrm{~K}$, the copper current density can exceed 1000 $\mathrm{A} / \mathrm{mm}^{2}$. For comparison, the copper current density in a $\mathrm{Nb}_{3} \mathrm{Sn}$ laboratory magnet is less than $300 \mathrm{~A} / \mathrm{mm}^{2}$. Ishiyama, et al., worked out general equations to estimate the optimal stabilizer cross section for a SMES magnet operated at 50 K [133]. Regarding high-field coils, simulation study by Uglietti and Marinucci has shown that a moderate increase in copper cross section coupled with a sensitive quench detection and a short discharge time constant are sufficient to passively protect a 4-T/40-mm bore magnet [134]. For large coils the copper cross section should be further increased if a passive protection system (shunt resistors with diodes) is used.

Active quench protection often uses heaters. When a quench is detected, the heaters placed within the winding are activated, either by an external power supply or by a portion of the stored energy, quickly driving a large volume of the winding into the normal state. This normal-state volume is generally designed to dissipate the entire stored energy with a limited temperature rise. The quench-inducing heater will be necessary for the HTS coil because of its low NZPV. Dixon et al. used a heater system in combination with diodes and resistors to protect a 900-MHz NMR 


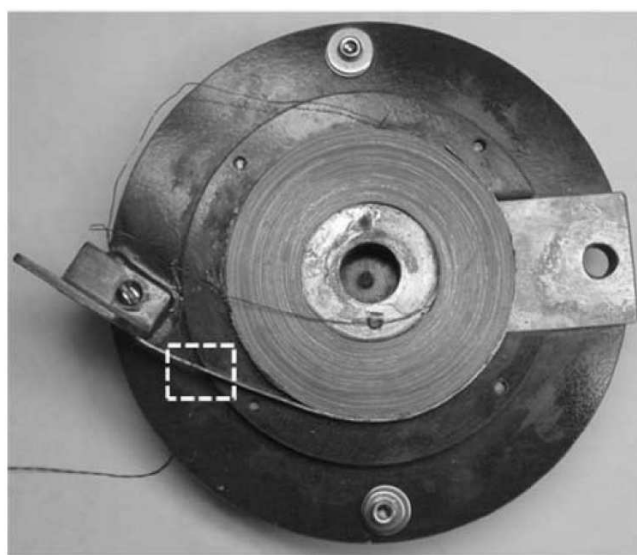

(a)

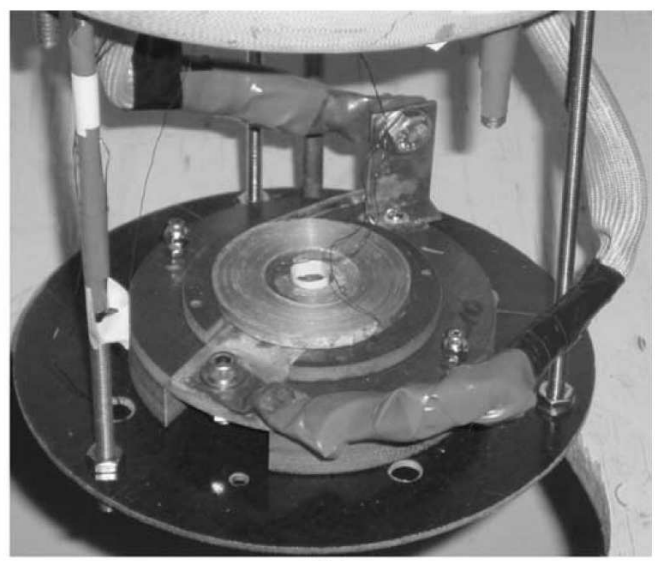

(b)

Figure 11 (a) No-Insulation (NI) HTS single pancake test coil; (b) Test coil mounted in the LHe experimental setup. Reprinted with permission from Hahn et al. Applied Superconductivity, IEEE Transactions on 22 4302405 [144], Copyright 2012, IEEE.

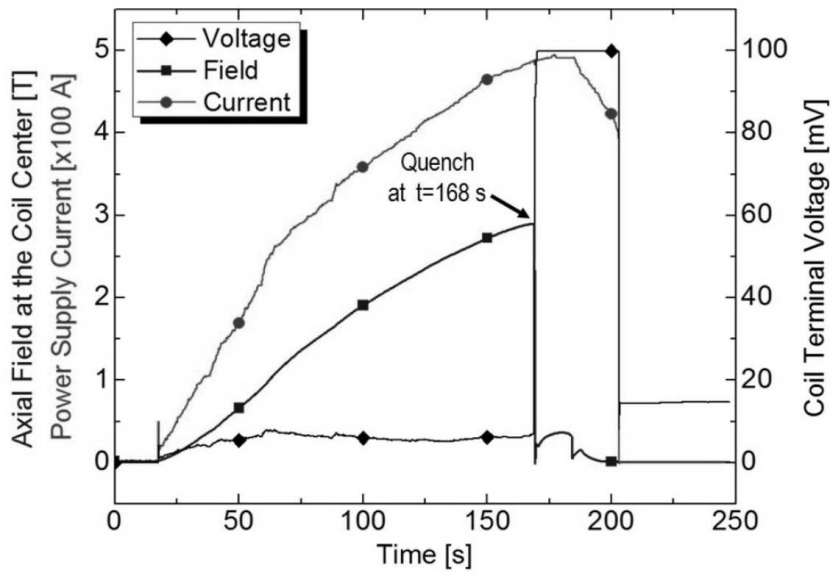

Figure 12 Over-current test results of an NI HTS test coil at $4.2 \mathrm{~K}$. Reprinted with permission from Hahn et al. Applied Superconductivity, IEEE Transactions on 224302405 [144], Copyright 2012, IEEE.

magnet comprising $\mathrm{NbTi}$ and $\mathrm{Nb}_{3} \mathrm{Sn}$ coils [135]. A heater-based active protection is more complicated to implement, both in design and construction, compared with a passive protection, but it is mandatory, especially for large HTS magnets. A heater-based quench protection system was tested on a Bi2212 insert coils [136]: the experiment shows that this solution is compatible in terms of response time with the protection system of the outer LTS coils. General considerations about protection heaters for coated conductor coils are presented in [137] where heaters are designed for various coil sizes and operating temperature: in general a dense array of heaters is effective for all the cases presented in the paper. A practical application of these considerations is presented in [138] for the protection of a 32$\mathrm{T}$ magnet under construction at the NHMFL (see Section 4): quench tests of the double pancake modules were carried out to demonstrate that the $32 \mathrm{~T}$ magnet would be safely protected.

\subsubsection{No-Insulation (NI) Coils}

In a very different approach to quench protection, turn-to-turn electrical insulation is removed, completely or partially, to enable the current to flow into the adjacent turns and thereby keep the hot spot from overheated. An $\mathrm{Nb}_{3} \mathrm{Sn}$ layer-wound coil with partial insulation (layers insulated, no turn-to-turn insulation) was tested in 1986 [139] and the first no-insulation (NI) coil in 1999 [140]. This NI coil consisted of an NbTi cable-in-conduit conductor, which was directly soldered to the coil mandrel to form a detector magnet; in case of quench the current is shunted 
(a)

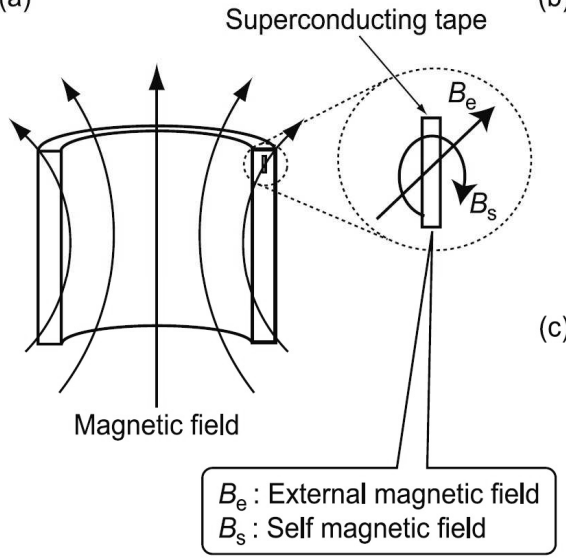

(b)

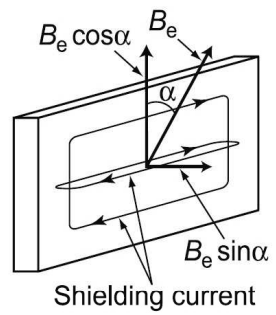

(c)

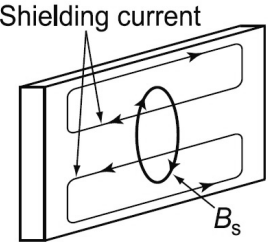

Figure 13 Magnetic fields and shielding currents in superconducting tape: (a) external magnetic field $B_{\mathrm{e}}$ and selfmagnetic field $B_{\mathrm{s}}$ for superconducting tape, (b) shielding (magnetization) current against $B_{\mathrm{e}}$, and (c) shielding current against $B_{\mathrm{s}}$. Reprinted from [148].

through the steel conduit. A new detector magnet based on the same design principle was constructed in 2008 [141]. The NI winding technique has also been applied to HTS double-pancake coils wound with Bi2223 and RE123 [142]. Tests at $77 \mathrm{~K}$ with these coils demonstrated that the NI coils can withstand over 2.5 times the coil critical current without burning, while insulated coil are very quickly damaged as soon as the critical current is exceeded. Investigation on NZPV was carried out with distributed thermocouples and voltage taps [143].

With coated conductors measurement at $4.2 \mathrm{~K}$ of a single pancake (25-mm i.d., 53-mm o.d., see Fig. 11) confirmed an exceptional tolerance to over-current: the pancake fully quenched at $486 \mathrm{~A}$ and such a high current was maintained for over $30 \mathrm{~s}$ [144] (see Fig. 12). The tape connecting the coil to the copper electrode burned out (see dashed region in Fig. 11), but measurements at $77 \mathrm{~K}$ showed that the coil itself was not damaged. Therefore the authors have suggested that a non-insulated coil should be regarded as a self-protecting coil, which does not need neither active nor passive protection, i.e. the stored energy can be dissipated in the coil winding without any other circuit or device. An additional benefit is that by removing the insulation and possibly decreasing the stabilizer thickness, the overall coil current density can be further increased.

The main drawback of the NI winding technique is a charging delay time when an NI coil is energized. An NI coil may be modelled with an equivalent LR electric circuit with L given by the coil inductance and $\mathrm{R}$ by the turn-to-turn resistance [145]. In the layer-wound coil the delay time constant becomes too long to make the coil impractical [146], unless the coil incorporates partial-insulation winding technique [139, 147].

\subsection{Field quality}

The field quality is a very important feature of a superconducting magnet. Field quality includes two aspects: temporal stability; and spatial homogeneity (about the magnet centre). The field quality for NMR and MRI magnets are much more stringent than that for standard laboratory magnets. For example, an NMR magnet may require a temporal field stability of better than $0.01 \mathrm{ppm} / \mathrm{h}$, which, for a $20 \mathrm{~T}$ magnet, corresponds to a variation of $1.5 \mathrm{mT}$ per year. The tolerance on coil geometry is a key parameter for meeting the spatial homogeneity requirements. This is one reason why layer-wound construction is preferred to pancake construction for MRI and NMR magnets, even with tape conductors. 


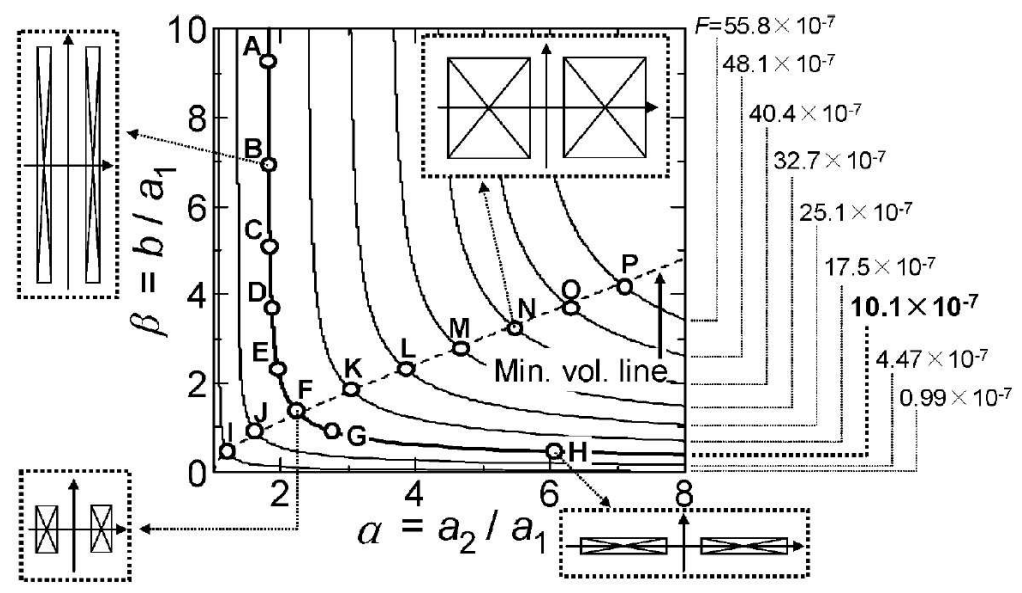

Figure 14 Lines of constant shape factor $F$ as a function of the coil geometry: $2 a_{1}$ is the bore diameter, $2 a_{2}$ the coil diameter and $2 b$ the coil height. Coils on the same line generate the same central magnetic field, if the overall current density and the inner diameter are the same. Coils on the minimum volume line generate a central magnetic field with minimum use of superconducting wire. Reprinted with permission from Yanagisawa et al. Applied Superconductivity, IEEE Transactions on 20 744-7 [156], Copyright 2011, IEEE.

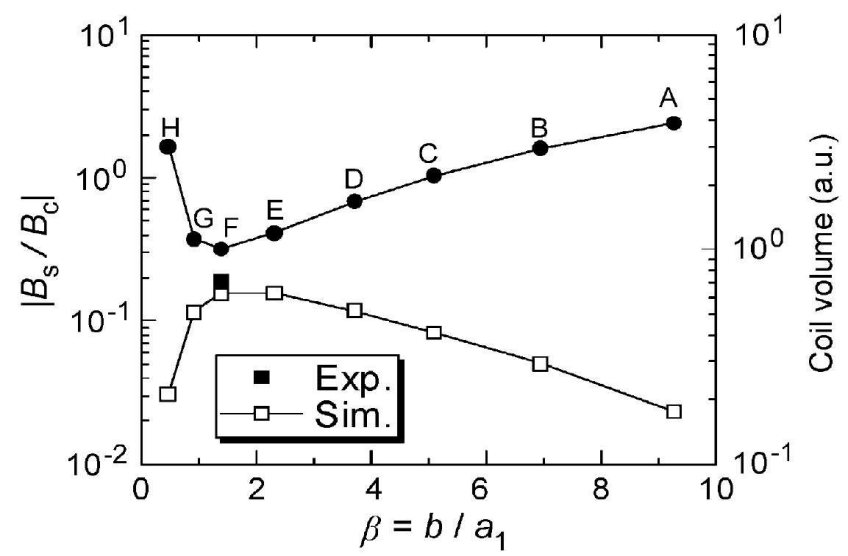

Figure 15 The ratio between the screening field and the design central field as a function of the coil height normalized to the bore diameter. Reprinted with permission from Yanagisawa et al. Applied Superconductivity, IEEE Transactions on 20 744-7 [156], Copyright 2011, IEEE.

The field quality of a magnet is degraded, among other factors, by the magnetization of the magnet's superconductor. Based on Bean's model, the magnetization is a result of a screening current induced in the superconductor in response to a time-varying field. In a tape-wound magnet, the radial component of a magnetic field will induce large superconducting screening current loops in the tape [148]. The situation is schematically shown in Fig. 13. The magnetic field generated by the screening currents is also called shielding field, field error or magnetisation.

The screening-current field (SCF) is responsible for: 1) field reduction with respect to the design value; 2) remnant field after the magnet has been discharged; 3) temporal field drift at constant operating current (or in persistent mode) by flux creep. The SCF varies along the magnet axis, thus affecting also the field homogeneity. Some of the very first superconducting solenoid magnets built in the 1960 s were wound either with $\mathrm{V}_{3} \mathrm{Ga}$ tape or $\mathrm{Nb}_{3} \mathrm{Sn}$ tape, whose geometry and architecture are similar to those of modern coated conductors. Indeed those magnets exhibited a reduced central field, a sizable remnant field after discharge, and large temporal field drift (at constant operating current) [149-151]. Since the beginning of the 1970s multifilamentary wires ( $\mathrm{NbTi}$ and $\left.\mathrm{Nb}_{3} \mathrm{Sn}\right)$ have become the standard in the industry. Because of the fine filaments (2 to $100 \mu \mathrm{m})$ the screening current loops are very small, making their SCF negligible. Nevertheless for NMR and MRI magnets, filaments should be as fine as possible 


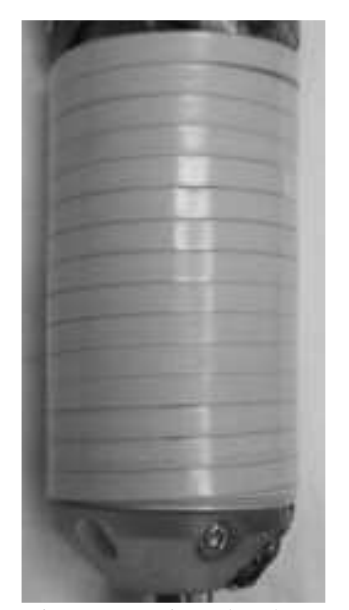

Figure 16 RE123 test coil fron NHMFL generating 4.2 T in a background field of $31.2 \mathrm{~T}$. Reprinted with permission from Trociewitz et al., Applied Physics Letters 99202506 [75]. Copyright 2011, AIP Publishing LLC.

(typical $\mathrm{Nb}_{3} \mathrm{Sn}$ Bronze Route wires for NMR magnets have filaments diameters betwen $2 \mu \mathrm{m}$ and $5 \mu \mathrm{m}$ ). The detrimental effects of SCF on field quality were observed in the first decade of this century, when Bi2223 tapes were used to build inserts for NMR magnets [152, 153]. In [154] and [155] the SCF of the Bi2223 insert was found to be responsible for reduction in the total magnetic field, a large gradient in the harmonic components, and large temporal drift (field decay). Although Bi2223 tape, unlike coated conductor tape, consists of many filaments, because the filaments are embedded in silver matrix and thus electromagnetically coupled, its SCF is large.

SCF will be even larger in coated conductor, which basically consists of a single monofilament (the superconducting layer) of width in the range 3-12 mm: this is about three orders of magnitude larger than the finest filaments in $\mathrm{Nb}_{3} \mathrm{Sn}$ wires. It follows that the detrimental effects of SCF on field quality should also be larger in the same order. A detailed calculation of SCF in coated conductor coils (see Fig. 14) was carried out at LHe temperature [156]. It was found that the centre field ratio of the SCF to the coil is maximum for a minimum-volume coil, whereas it is much lower for short-and-thick (pancake) coils and long-and-thin coils. For a fixed aspect ratio, for example, a minimumvolume coil, the ratio first increases with coil volume, reaches a maximum, and then decreases (see Fig. 15). Moreover, for a fixed coil shape, SFC is proportional to the ratio of tape width to coil i.d. [157]. Of course, minimum-volume coil is generally unsuitable to meet high spatial field homogeneity requirements.

The detrimental effects of SFC on field for an all-coated-conductor 1.3 GHz NMR magnet have been evaluated numerically, [158]: at the centre the SFC would reduce the strength by $\sim 8 \%$ and deteriorate the spatial homogeneity. Even if NbTi correction coils are placed at magnet ends, they are ineffective to improve the field homogeneity.

Field reduction and remnant field were measured for coils of different aspect ratios [159]; measurement and computation ([157]) agree quite well. The field temporal stability in a small coil of the same aspect ratio as that of a nominal-size NMR coil was measured; the small coil was found to have a temporal drift of $10 \mathrm{ppm} / \mathrm{h}$, clearly much greater than that for a typical NMR operation [159]. One technique proposed recently to shim SFC-induced field impurity is a set of persistent-mode shim coils prepared from RE123 [160].

Despite a report of a superconducting joint between coated superconductors (see Section 3.1.5), a persistent-mode NMR magnet of coated conductor could still be difficult because of the temporal drift due to the screening currents. Various techniques have been proposed to reduce the amplitude of the screening currents. These possible techniques include: multifilamentary coated conductors (striated coated conductors, see Sec. 2.4) would reduce SCF inversely to the number of filaments, for example, with 10 filaments, SCF would be reduced by 10; the so-called overshooting technique [161], though not really useful for the NMR magnet, reverses a current sweep, as small as $1 \%$ of the peak 
Table 4 Main parameters of the ongoing high-field projects based on RE123

$\begin{array}{lcccccc} & 35.4 \mathrm{~T} & 1.3 \mathrm{GHz} & 32 \mathrm{~T} & 24 \mathrm{~T} & 25 \mathrm{~T} \text { CSM } & 100 \mathrm{~T} \\ & \text { NHMFL } & \text { MIT } & \text { NHMFL } & \text { NIMS } & \text { HFLSM } & \text { MIT } \\ \text { Target Field }(T) & 35.4 & 30.5 & 32 & 24 & 25.6 & 100 \\ \text { HTS Field }(T) & 4.2 & 11.7 & 17 & 7.6 & 11.5 & 100 \\ \text { RE123 coil Field }(T) & 4.2 & 18.8 & 17 & 7.6 & 11.5 & 100 \\ \text { No. } \text { RE123 Sections } & 1 & 3 & 2 & 1 & 1 & 39 \\ \text { Inner Diameter }(\mathrm{mm}) & 14 & 91 & 40 & 50 & 96 & 20 \\ \text { Outer Diameter }(\mathrm{mm}) & 38 & 204 & 232 & 112.4 & 280 & 5600 \\ \text { Winding Technology } & \text { LW } & \text { DP } & \text { DP } & \text { LW } & \text { LW } & \text { DP } \\ \text { Operating Current } & 196 & 250 & 180 & 360 & 135 & 4 \times 600 \\ \text { Winding Curr. Dens. }\left(\mathrm{A} / \mathrm{mm}^{2}\right) & 320 & 546 & 197 / 176 & 262 & 110.8 & 520.8 \\ \text { Copper Curr. Dens. }\left(\mathrm{A} / \mathrm{mm}^{2}\right) & 1200 & 2042^{*} & 439 & 960 & - & - \\ \text { Max. Hoop Stress }(\mathrm{MPa}) & 340 & 680 & 450 & 450 & 405 & 398 \\ \text { Total RE123 }(\mathrm{m}) & 96 & 11 \text { '590 } & 9{ }^{2} 379 & 515 & 17 \text { '591 } & 12 \text { '367’000 } \\ \text { Status } & \text { Tested } & \text { In Prod. } & \text { In Prod. } & \text { Tested } & \text { Concept } & \text { Concept }\end{array}$

current, to impede SCF variation; apply a field parallel to the tape wide surface with copper coils that sandwich a coated conductor coil [162].

\section{Overview on Ongoing High-Field DC Magnet projects based on RE123}

The progress of high-field magnets has tracked closely the advancement of superconductors and materials science. It has been propelled by new frontiers of science and science-driven innovation in which a magnetic field plays a key role. The trends and driving forces for these ambitious high-field magnets are summarized in a 2013 report by the National Academy of Sciences [163]. Among new ideas, recommendations, future technological objectives, the report recommends the construction of a $40 \mathrm{~T}$ all-superconducting $\mathrm{DC}$ magnet that should motivate research institutions in the world active in superconducting magnet technology.

In this section we present selected steady (DC) all-RE123 high-field magnets, the main design parameters of which are given in Table 4.

The highest magnetic field ever achieved, $35.4 \mathrm{~T}$, was obtained with a 4.2-T insert wound with 96-m long RE123 in a 31.2-T resistive outsert at the NHMFL (Fig. 16) [75]. The RE123 insert incorporates an innovative insulation material of non-bonding thin polyester film that prevents conductor degradation during cooling (see Sections 2.5.3 and 3.1.3).

An important project that has been upgraded along the course of the years is a 1.3-GHz (30.5-T) NMR magnet under construction at the MIT Francis Bitter Magnet Laboratory [164]. The magnet is particularly interesting, and challenging, because it fully capitalizes the high-field potential of RE123, making the RE123 insert, despite stringent NMR-quality field requirements, contribute $800 \mathrm{MHz}$, over $60 \%$ of the total frequency (and field). Upon successful completion, this magnet will become a power tool for advancement in biomedical sciences [163]. This 500-MHz LTS/800-MHz RE123 combination superseded a 600-MHz LTS/700-MHz HTS (Bi2223/RE123) combination [165], which had earlier replaced yet another combination of a 700-MHz LTS/600 MHz HTS (Bi2223/RE123) [166]. Two of the most remarkable features of this design are related to the insulation strategy and field quality. In fact a noinsulation (NI) winding technique is adopted in order to make the $800-\mathrm{MHz}$ RE123 insert self-protecting without 


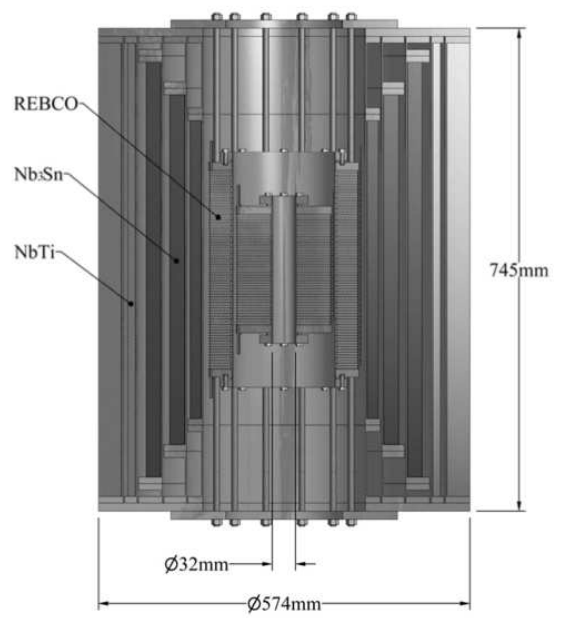

Figure 17 Design of a superconducting $32 \mathrm{~T}$ magnet with RE123 high field coils, from NHMFL. Reprinted from [170].

adding extra $\mathrm{Cu}$ stabilizer, compact, and mechanically robust. Despite its rather short overall winding length, its computed field quality, in the absence of SCF, is maintained with inside-notched central DP coils [164].

Another high-field NMR system is being developed at NIMS, Tsukuba, in Japan, in which the goal is to go beyond 1 $\mathrm{GHz}[167,168]$. The project is going through several intermediate stages to develop RE123 magnet technology. Currently their focus is on a $600 \mathrm{MHz}$ RE123 system. For this purpose they tested in 2011 a short layer-wound 7.6-T RE123 insert in a background LTS field of 17.2 T [169]. An interesting feature introduced in this test coil is a resistive joint connecting the two 250-m long RE123 sections. The splice, embedded within the coil winding, was operated successfully up to $321 \mathrm{~A}$. Although the coil design was quite conservative in regard to a maximum stress (460 MPa) its copper current density was quite ambitious $\left(960 \mathrm{~A} / \mathrm{mm}^{2}\right)$, requiring a reliable protection system.

In terms of laboratory high-field magnets targeting materials science and solid-state physics research, a 32-T magnet project at NHMFL deserves a special citation: today this project is one of the most advanced, incorporating all-LTS and HTS coils in a unique high-field facility (Fig. 17) [111, 170]. The program has a stated double purpose of enabling unprecedented high-field research activities and demonstrating HTS-based magnet technologies. The design parameters for this magnet, aimed to serve the user for 20 years, focuses on modularity and generous operating margins. The hoop-stress is limited to $450 \mathrm{MPa}$ on RE123 conductor and copper current density to $440 \mathrm{~A} / \mathrm{mm}^{2}$ to ease protection requirements. These rather conservative choices, taken to ensure reliability and longevity, pose challenge, particularly for RE123 insert. The total RE123 conductor length is almost $10 \mathrm{~km}$, configured into 56 double-pancake coils, each wound with special insulation and reinforcement mechanism, and protected [171].

It is interesting to note that for some of these projects there has been a shift from hybrid Bi2223-RE123 coil formation toward all-RE123 formation [164, 166, 172-174]. This shift may reflect the RE123's steady performance improvement in electromagnetic properties and its inherent mechanical strength advantage over Bi2223. Based on the design parameters listed in Table 4, it appears that the adopted stress management strategies even under severe operating conditions are consistent with the well-known RE123 properties. On the other hand, there still remains a wide range of protection strategies, from the self-protecting feature of the NI winding technology to the traditional protection techniques for insulated winding technology. This protection issue will certainly be subject of deeper investigations and debate in the near future when these projects will enter in their critical production and testing phases. 
In parallel to the high-field NMR project the High Field Laboratory for Superconducting Materials (HFLSC) in Sendai is also planning an upgrade to their $18 \mathrm{~T}$ cryogen-free superconducting magnet (CSM). The proposed field target of $25 \mathrm{~T}[172,173]$ could be achieved with a 14-T LTS outsert and a single RE123 insert. This RE123 coil also uses quite relaxed parameters, and combined with its large inner bore (96 mm winding diameter), the coil will require RE123 conductor in excess of $17 \mathrm{~km}$.

As a final closing citation in this section dedicated to high-magnetic field systems, a special note is reserved to a 100 T DC magnet proposed recently by Iwasa and Hahn [112]. Their design in fact pushes to the extreme limit all aspects of the RE123 technology and magnet design developed to date. The technological effort will be comparable with those of LHC ATLAS and ITER TF magnets: the 100-T magnet, almost 17-m tall and nearly 6-m outer diameter, stores more than $120 \mathrm{GJ}$ of magnetic energy. It comprises 39 nested all-RE123 coils, assembled from more than 14'000 double-pancake coils requiring a total of more than 12'000 km of 12-mm wide RE123 tape. One of the main enabling ingredients for this ambitious magnet is the RE123 conductor. With its unique electromagnetic properties and mechanical strength, RE123 seems at present the only suitable superconductor. A 100-T DC superconducting magnet, perhaps the Holy Grail of a continuous (DC) magnetic field, is an example of an innovative, and ambitious, design that in order to convert to reality requires a further superconducting magnet technology R\&D effort, and surely of an international scale, like ITER and LHC.

\section{Conclusions}

Currently, because of its current-carrying capacity and, equally importantly, high mechanical strength, RE123 is the only high-temperature superconductor applicable to high-field magnets. It is unlikely that even continued progress in Bi2212 and Bi2223 conductors will able to resolve their inherent disadvantage in mechanical strength vs. RE123. To fully capitalize on the remarkable properties of RE123, a wide range of techniques has been developed and some already successfully deployed. Despite many challenging issues that confront RE123, and the magnet, its demanding and inspiring partner, together they are poised to break through into a new brave high-field world, even unimagined at the beginning of the HTS era. We strongly believe that this new high-field world, where HTS is unquestionably the enabling technology, will lead to major discoveries and development in many areas of physics, biology, chemistry, materials, medicine, and engineering, possibly even entirely original studies that would have been impossible or even inconceivable.

\section{Acknowledgements}

CS acknowledge the financial support from the Swiss National Science Foundation (Grant No. PP00P2_144673 and 51NF40-144613).

\section{References}

[1] Wu M K, Ashburn J R, Torng C J, Hor P H, Meng R L, Gao L, Huang Z J, Wang Y Q and Chu C W 1987 Superconductivity at $93-\mathrm{K}$ in a New Mixed-Phase Y-Ba-Cu-O Compound System at Ambient Pressure Phys Rev Lett $\mathbf{5 8}$ 908-10

[2] Maeda H, Tanaka Y, Fukutomi M and Asano T 1988 A New High-Tc Oxide Superconductor without a Rare-Earth Element Jpn J Appl Phys 227 L209-L10

[3] Larbalestier D C, Jiang J, Trociewitz U P, Kametani F, Scheuerlein C, Dalban-Canassy M, Matras M, Chen P, Craig N C, Lee P J and Hellstrom E E 2014 Isotropic round-wire multifilament cuprate superconductor for generation of magnetic fields above 30 T Nature Materials 13 375-81 
[4] Iijima Y, Tanabe N, Kohno O and Ikeno Y 1992 Inplane Aligned Yba2cu3o7-X Thin-Films Deposited on Polycrystalline Metallic Substrates Applied Physics Letters 60 769-71

[5] Graser S, Hirschfeld P J, Kopp T, Gutser R, Andersen B M and Mannhart J 2010 How grain boundaries limit supercurrents in high-temperature superconductors Nature Physics 6 609-14

[6] Hilgenkamp H and Mannhart J 2002 Grain boundaries in high-T superconductors Rev. Mod. Phys. 74485

[7] Lehner T 2012 Investigation of HTS Materials for Electric Power Equipment. (Workshop on Present, Status Future Perspectives of Hts Power Applications, Paris http://www.superpowerinc.com/system/files/2012_0829+CIGRE+WS+Pres_Lehner_HTS+Materials+FINAL.pdf)

[8] Goyal A, Norton D P, Kroeger D M, Christen D K, Paranthaman M, Specht E D, Budai J D, He Q, Saffian B, List F A, Lee D F, Hatfield E, Martin P M, Klabunde C E, Mathis J and Park C 1997 Conductors with controlled grain boundaries: An approach to the next generation, high temperature superconducting wire Journal of Materials Research 12 2924-40

[9] Groves J R, Arendt P N, Kung H, Foltyn S R, DePaula R F, Emmert L A and Storer J G 2001 Texture development in IBAD MgO films as a function of deposition thickness and rate Applied Superconductivity, IEEE Transactions on $112822-5$

[10] Rupich M W, Zhang W, Li X, Kodenkandath T, Verebelyi D T, Schoop U, Thieme C, Teplitsky M, Lynch J, Nguyen N, Siegal E, Scudiere J, Maroni V, Venkataraman K, Miller D and Holesinger T G 2004 Progress on MOD/RABiTSTM 2G HTS wire Physica C: Superconductivity 412-414 877-84

[11] Nagaishi T, Shingai Y, Konishi M, Taneda T, Ota H, Honda G, Kato T and Ohmatsu K 2009 Development of REBCO coated conductors on textured metallic substrates Physica C: Superconductivity 469 1311-5

[12] Rupich M, Li X, Thieme C, Sathyamurthy S, Fleshler S, Tucker D, Thompson E, Schreiber J, Lynch J, Buczek D, DeMoranville K, Inch J, Cedrone P and Slack J 2010 Advances in second generation high temperature superconducting wire manufacturing and R\&D at American Superconductor Corporation Superconductor Science and Technology 23014015

[13] Rupich M W, Li X, Sathyamurthy S, Thieme C L H, DeMoranville K, Gannon J and Fleshler S 2013 Second Generation Wire Development at AMSC Applied Superconductivity, IEEE Transactions on 23 6601205

[14] Suo H, Zhao Y, Liu M, Ma L, He D, Zhang Y and Zhou M 2007 Preparation of Cube Textured Ni5W/Ni9W Composite Substrate for YBCO Coated Conductors Applied Superconductivity, IEEE Transactions on 17 3420-3

[15] Oh S S, Ha H S, Kim H S, Ko R K, Song K J, Ha D W, Kim T H, Lee N J, Youm D, Yang J S, Kim H K, Yu K K, Moon S H, Ko K P and Yoo S I 2008 Development of long-length SmBCO coated conductors using a batch-type reactive co-evaporation method Superconductor Science and Technology 21034003

[16] Igarashi M, Kakimoto K, Hanyu S, Tashita C, Hayashida T, Hanada Y, Fujita S, Morita K, Nakamura N, Sutoh Y, Kutami H, Iijima Y and Saitoh T 2010 Remarkable progress in fabricating RE123 coated conductors by IBAD/PLD technique at Fujikura Journal of Physics: Conference Series 234022016

[17] Dong L and Srolovitz D J 1998 Texture development mechanisms in ion beam assisted deposition Journal of Applied Physics $\mathbf{8 4} 5261-9$

[18] Igarashi M 2009 RE123 Coated Conductors Fujikura Technical Review No.38 http://www.fujikura.co.jp/eng/rd/gihou/backnumber/pages/1198117_4995.html

[19] Xiong X, Kim S, Zdun K, Sambandam S, Rar A, Lenseth K P and Selvamanickam V 2009 Progress in High Throughput Processing of Long-Length, High Quality, and Low Cost IBAD MgO Buffer Tapes at SuperPower Applied Superconductivity, IEEE Transactions on 19 3319-22

[20] Lee S, Petrykin V, Molodyk A, Samoilenkov S, Kaul A, Vavilov A, Vysotsky V and Fetisov S 2014 Development and production of second generation high T-c superconducting tapes at SuperOx and first tests of model cables Supercond Sci Tech 27044022

[21] Usoskin A, Kirchhoff, Knoke J, Prause B, Rutt, Selskij and Farrell D E 2007 Processing of Long-Length YBCO Coated Conductors Based on Stainless Steel Tapes Applied Superconductivity, IEEE Transactions on $173235-8$

[22] Matias V, Rowley J, Coulter Y, Maiorov B, Holesinger T, Yung C, Glyantsev V and Moeckly B 2010 YBCO films grown by reactive co-evaporation on simplified IBAD-MgO coated conductor templates Superconductor Science and Technology 23014018

[23] Malozemoff A P, Fleshler S, Rupich M, Thieme C, Li X, Zhang W, Otto A, Maguire J, Folts D, Yuan J, Kraemer H P, Schmidt W, Wohlfart M and Neumueller H W 2008 Progress in high temperature superconductor coated conductors and their applications Superconductor Science and Technology 21 034005

[24] Selvamanickam V, Galinski G B, Carota G, DeFrank J, Trautwein C, Haldar P, Balachandran U, Chudzik M, Coulter J Y, Arendt P N, Groves J R, DePaula R F, Newnam B E and Peterson D E 2000 High-current $\mathrm{Y}-\mathrm{Ba}-\mathrm{Cu}-\mathrm{O}$ superconducting films by metal organic chemical vapor deposition on flexible metal substrates Physica C: Superconductivity 333 155-62 
[25] Watanabe T, Kuriki R, Iwai H, Muroga T, Miyata S, Ibi A, Yamada Y and Shiohara Y 2005 High rate deposition by PLD of YBCO films for coated conductors Applied Superconductivity, IEEE Transactions on 15 2566-9

[26] Usoskin A, Knoke J, Garcia-Moreno F, Issaev A, Dzick J, Sievers S and Freyhardt H C 2001 Large-area HTS-coated stainless steel tapes with high critical currents Applied Superconductivity, IEEE Transactions on $113385-8$

[27] Van Driessche I, Feys J, Hopkins S C, Lommens P, Granados X, Glowacki B A, Ricart S, Holzapfel B, Vilardell M, Kirchner A and Bäcker M 2012 Chemical solution deposition using ink-jet printing for YBCO coated conductors Superconductor Science and Technology 25065017

[28] Obradors X, Puig T, Ricart S, Coll M, Gazquez J, Palau A and Granados X 2012 Growth, nanostructure and vortex pinning in superconducting $\mathrm{YBa}_{2} \mathrm{Cu}_{3} \mathrm{O}_{7}$ thin films based on trifluoroacetate solutions Superconductor Science and Technology 25123001

[29] Christen H M and Eres G 2008 Recent advances in pulsed-laser deposition of complex oxides Journal of Physics: Condensed Matter 20264005

[30] Ma B, Koritala R E, Fisher B L, Uprety K K, Baurceanu R, Dorris S E, Miller D J, Berghuis P, Gray K E and Balachandran U 2004 High critical current density of YBCO coated conductors fabricated by inclined substrate deposition Physica C: Superconductivity 403 183-90

[31] Moon S H 2013 SuNAM developed new process named RCE-DR: the practical highest throughput process 11th European Conference on Applied Superconductivity EuCAS2013 www.ewh.ieee.org/tc/csc/global/issue26/moon.pdf

[32] Moon S H 2014 HTS Development and Industrialization at SuNAM. (1st Workshop on Accelerator Magnets in HTS, Hamburg, Germany: http://indico.cern.ch/event/308828/session/2/contribution/10/material/slides/1.pdf)

[33] MacManus-Driscoll J L, Foltyn S R, Jia Q X, Wang H, Serquis A, Civale L, Maiorov B, Hawley M E, Maley M P and Peterson D E 2004 Strongly enhanced current densities in superconducting coated conductors of $\mathrm{YBa}_{2} \mathrm{Cu}_{3} \mathrm{O}_{7-\mathrm{x}}+\mathrm{BaZrO}_{3}$ Nature materials 3 439-43

[34] Chen Y, Selvamanickam V, Zhang Y, Zuev Y, Cantoni C, Specht E, Paranthaman P, Aytug T, Goyal A and Lee D 2009 Enhanced flux pinning by $\mathrm{BaZrO}_{3}$ and $(\mathrm{Gd}, \mathrm{Y})_{2} \mathrm{O}_{3}$ nanostructures in metal organic chemical vapor deposited GdYBCO high temperature superconductor tapes Applied Physics Letters 94062513

[35] Gutierrez J, Llordes A, Gazquez J, Gibert M, Roma N, Ricart S, Pomar A, Sandiumenge F, Mestres N, Puig $\mathrm{T}$ and Obradors X 2007 Strong isotropic flux pinning in solution-derived $\mathrm{YBa}_{2} \mathrm{Cu}_{3} \mathrm{O}_{7-\mathrm{x}}$ nanocomposite superconductor films Nature Materials 6 367-73

[36] Matsushita T, Nagamizu H, Tanabe K, Kiuchi M, Otabe E S, Tobita H, Yoshizumi M, Izumi T, Shiohara Y, Yokoe D, Kato T and Hirayama T 2012 Improvement of flux pinning performance at high magnetic fields in $\mathrm{GdBa}_{2} \mathrm{Cu}_{3} \mathrm{O}_{\mathrm{y}}$ coated conductors with $\mathrm{BHO}$ nano-rods through enhancement of $\mathrm{B}_{\mathrm{c} 2}$ Superconductor Science and Technology 25125003

[37] Selvamanickam V, Yao Y, Chen Y, Shi T, Liu Y, Khatri N D, Liu J, Lei C, Galstyan E and Majkic G 2012 The low-temperature, high-magnetic-field critical current characteristics of $\mathrm{Zr}$-added $(\mathrm{Gd}, \mathrm{Y}) \mathrm{Ba}_{2} \mathrm{Cu}_{3} \mathrm{O}_{\mathrm{x}}$ superconducting tapes Superconductor Science and Technology 25125013

[38] Miura M, Maiorov B, Baily S A, Haberkorn N, Willis J O, Marken K, Izumi T, Shiohara Y and Civale L 2011 Mixed pinning landscape in nanoparticle-introduced $\mathrm{YGdBa}_{2} \mathrm{Cu}_{3} \mathrm{O}_{\mathrm{y}}$ films grown by metal organic deposition Physical Review B 83184519

[39] Choi S M, Lee J W, Shin G H, Lee J H, Hong G W, Moon S H and Yoo S I 2013 Characteristics of HighJ(c) GdBCO Coated Conductors Fabricated by the RCE-DR Process Ieee T Appl Supercon 238001004

[40] Selvamanickam V, Chen Y, Kesgin I, Guevara A, Shi T, Yao Y, Qiao Y, Zhang Y, Majkic G, Carota G, Rar A, Xie Y, Dackow J, Maiorov B, Civale L, Braccini V, Jaroszynski J, Xu A, Larbalestier D and Bhattacharya R 2011 Progress in Performance Improvement and New Research Areas for Cost Reduction of 2G HTS Wires Applied Superconductivity, IEEE Transactions on 21 3049-54

[41] Braccini V, Xu A, Jaroszynski J, Xin Y, Larbalestier D C, Chen Y, Carota G, Dackow J, Kesgin I, Yao Y, Guevara A, Shi T and Selvamanickam V 2011 Properties of recent IBAD-MOCVD coated conductors relevant to their high field, low temperature magnet use Superconductor Science and Technology 24035001

[42] Weijers H W 2009 High-temperature superconductors in high-field magnets. (PhD Thesis: University of Twente)

[43] Hahn S, Kim Y, Park D K, Kim K, Voccio J P, Bascunan J and Iwasa Y 2013 No-insulation multi-width winding technique for high temperature superconducting magnet Applied Physics Letters 103173511

[44] Obradors X and Puig T 2014 Coated conductors for power applications: materials challenges Supercond Sci Tech 27044033

[45] Rolando G 2013 Cable-in-conduit superconductors for fusion magnets. (PhD Thesis: University of Twente)

[46] Lu J, Choi E S, Kandel H, Abraimov D V and Markiewicz W D 2014 Hysteresis Loss of REBCO Conductor Up to 35 T Ieee T Appl Supercon 248200104

[47] Kesgin I, Majkic G and Selvamanickam V 2013 Fully filamentized HTS coated conductor via striation and selective electroplating Physica C: Superconductivity 486 43-50 
[48] Kuhn M, Schey B, Klarmann R, Biegel W, Stritzker B, Eisenmenger J and Leiderer P 1998 Patterning of YBCO thin films by ion implantation and magneto-optical investigations Physica C: Superconductivity 294 $1-6$

[49] Cobb C, Barnes P, Haugan T, Tolliver J, Lee E, Sumption M, Collings E and Oberly C 2002 Hysteretic loss reduction in striated YBCO Physica C: Superconductivity 382 52-6

[50] Duckworth R C, Paranthaman M P, Bhuiyan M S, List F A and Gouge M J 2007 AC Losses in YBCO Coated Conductor With Inkjet Filaments Applied Superconductivity, IEEE Transactions on 17 3159-62

[51] Ma Q Y, Dosanjh P, Wong A, Carolan J F and Hardy W N 1994 Inhibition patterning of oxide superconducting films with Si ion implantation Superconductor Science and Technology 7294

[52] Sunwong P, Higgins J S, Tsui Y, Raine M J and Hampshire D P 2013 The critical current density of grain boundary channels in polycrystalline HTS and LTS superconductors in magnetic fields Superconductor Science and Technology 26095006

[53] Mondonico G, Seeber B, Senatore C, Flükiger R, Corato V, De Marzi G and Muzzi L 2010 Improvement of electromechanical properties of an ITER internal tin $\mathrm{Nb}_{3} \mathrm{Sn}$ wire Journal of Applied Physics 108093906

[54] Welp U 1992 Effect of uniaxial stress on the superconducting transition in $\mathrm{YBa}_{2} \mathrm{Cu}_{3} \mathrm{O}_{7}$ Phys. Rev. Lett. 69 2130

[55] van der Laan D C, Abraimov D, Polyanskii A A, Larbalestier D C, Douglas J F, Semerad R and Bauer M 2011 Anisotropic in-plane reversible strain effect in $\mathrm{Y}_{0.5} \mathrm{Gd}_{0.5} \mathrm{Ba}_{2} \mathrm{Cu}_{3} \mathrm{O}_{7-\delta}$ coated conductors Superconductor Science and Technology 24115010

[56] Sugano M, Machiya S, Oguro H, Sato M, Koganezawa T, Watanabe T, Shikimachi K, Hirano N, Nagaya S, Izumi T and Saitoh T 2012 The effect of the 2D internal strain state on the critical current in GdBCO coated conductors Superconductor Science and Technology 25054014

[57] van der Laan D C, Lu X F and Goodrich L F 2011 Compact GdBa2Cu3O7- $\delta$ coated conductor cables for electric power transmission and magnet applications Superconductor Science and Technology 24042001

[58] van der Laan D C, Ekin J W, Douglas J F, Clickner C C, Stauffer T C and Goodrich L F 2010 Effect of strain, magnetic field and field angle on the critical current density of $\mathrm{YBa}_{2} \mathrm{Cu}_{3} \mathrm{O}_{7-\delta}$ coated conductors Superconductor Science and Technology 23072001

[59] Sugano M, Shikimachi K, Hirano N and Nagaya S 2010 The reversible strain effect on critical current over a wide range of temperatures and magnetic fields for YBCO coated conductors Superconductor Science and Technology 23085013

[60] Kinoshita K HTS development and industrialization at Sumitomo (1st Workshop on Accelerator Magnets in HTS, Hamburg, Germany: http://indico.cern.ch/event/308828/session/2/contribution/9/material/slides/1.pdf)

[61] Cheggour N, Ekin J W, Xie Y Y, Selvamanickam V, Thieme C L H and Verebelyi D T 2005 Enhancement of the irreversible axial-strain limit of $\mathrm{Y}-\mathrm{Ba}-\mathrm{Cu}-\mathrm{O}$-coated conductors with the addition of a $\mathrm{Cu}$ layer Applied Physics Letters 87212505

[62] Shin H-S, Kim K-H, John R, Kim T-Y, Ko R-K and Oh S-S 2005 The strain effect on critical current in YBCO coated conductors with different stabilizing layers Superconductor Science and Technology 18 S364

[63] Osamura K, Machiya S, Ochiai S, Osabe G, Yamazaki K and Fujikami J 2013 High Strength/High Strain Tolerance DI-BSCCO Tapes by Means of Pre-Tensioned Lamination Technique Applied Superconductivity, IEEE Transactions on $\mathbf{2 3} 6400504$

[64] Shin H-S and Dedicatoria M 2012 Variation of the strain effect on the critical current due to external lamination in REBCO coated conductors Superconductor Science and Technology 25054013

[65] Shin H-S and Dedicatoria M 2013 Intrinsic strain effect on critical current in Cu-stabilized GdBCO coated conductor tapes with different substrates Superconductor Science and Technology 26055005

[66] Uglietti D, Seeber B, Abächerli V, Carter W L and Flükiger R 2006 Critical currents versus applied strain for industrial Y-123 coated conductors at various temperatures and magnetic fields up to $19 \mathrm{~T}$ Superconductor Science and Technology 19 869-72

[67] Usoskin A 2011 Physical principles and applications of inductive HTS shielded fault current limiting devices with suppressed cryogenic losses 10th EPRO Conference, Tallahassee, US http://mydocs.epri.com/docs/publicmeetingmaterials/1110/7TNRSL46577/02\% 20\% 20Physical\% 20Principles \% 20and \% 20Applications\% 20of\% 20Inductive\% 20HTS\% 20Shielded \% 20 Fault\% 20Current\% 20Limiting\% 20Devices\% 20(Usoskin).pdf

[68] Matias V, Jung Y and Sheehan C 2010 Progress in Reactive Co-Evaporation on IBAD Advanced Cables and Conductors Program Review, Alexandria, US http://www.htspeerreview.com/pdfs/presentations/day \% 202/2G/2_2G_Progress_in_Reactive_CoEvaporation_on_IBAD.pdf

[69] Mbaruku A L and Schwartz J 2008 Fatigue behavior of Y-Ba-Cu-O/Hastelloy-C coated conductor at $77 \mathrm{~K}$ Ieee T Appl Supercon 18 1743-52

[70] Sugano M, Yoshida Y, Hojo M, Shikimachi K, Hirano N and Nagaya S 2008 Two different mechanisms of fatigue damage due to cyclic stress loading at $77 \mathrm{~K}$ for MOCVD-YBCO-coated conductors Supercond Sci Tech 21054006 
[71] Shin H S and Dedicatoria M J 2011 Mechanical and transport properties of IBAD/EDDC-SmBCO coated conductor tapes during fatigue loading Cryogenics $\mathbf{5 1} 237-40$

[72] Yanagisawa Y, Nakagome H, Takematsu T, Takao T, Sato N, Takahashi M and Maeda H 2011 Remarkable weakness against cleavage stress for YBCO-coated conductors and its effect on the YBCO coil performance Physica C: Superconductivity 471 480-5

[73] Maeda H and Yanagisawa Y 2014 Recent Developments in High-Temperature Superconducting Magnet Technology (Review) Applied Superconductivity, IEEE Transactions on 244602412

[74] van der Laan D C, Ekin J W, Clickner C C and Stauffer T C 2007 Delamination strength of YBCO coated conductors under transverse tensile stress Superconductor Science and Technology 20 765-70

[75] Trociewitz U, Canassy M, Hannion M, Hilton D, Jaroszynski J, Noyes P, Viouchkov Y, Weijers H and Larbalestier D $201135.4 \mathrm{~T}$ field generated using a layer-wound superconducting coil made of $(\mathrm{RE}) \mathrm{Ba}_{2} \mathrm{Cu}_{3} \mathrm{O}_{7-\mathrm{x}}(\mathrm{RE}=$ rare earth$)$ coated conductor Applied Physics Letters 99202506

[76] Takematsu T, Hu R, Takao T, Yanagisawa Y, Nakagome H, Uglietti D, Kiyoshi T, Takahashi M and Maeda H 2010 Degradation of the performance of a YBCO-coated conductor double pancake coil due to epoxy impregnation Physica C: Superconductivity $\mathbf{4 7 0} 4$

[77] Yanagisawa Y, Sato K, Piao R, Nakagome H, Takematsu T, Takao T, Kamibayashi H, Takahashi M and Maeda H 2012 Removal of degradation of the performance of an epoxy impregnated YBCO-coated conductor double pancake coil by using a polyimide-electrodeposited YBCO-coated conductor Physica C: Superconductivity 476 19-22

[78] Sato K, Matsuda T, Yanagisawa Y, Nakagome H, Kamibayashi H, Uchida A, Takahashi M and Maeda H 2013 The Performance of a Practical Size Epoxy Impregnated Pancake Coil Wound With a Polyimide Electro-Deposited (PIED) YBCO-Coated Conductor Applied Superconductivity, IEEE Transactions on 23 4602504

[79] Maeda H, Takahashi M, Yanagisawa Y, Nakagome H, Oobuchi K and Kamibayashi H 2013 Coated hightemperature superconducting wire and high-temperature superconducting coil including the same European Patent Application 12189570.0, filed October 23, 2012, and published May 01, 2013

[80] Majkic G, Galstyan E, Zhang Y and Selvamanickam V 2013 Investigation of Delamination Mechanisms in IBAD-MOCVD REBCO Coated Conductors Applied Superconductivity, IEEE Transactions on 236600205

[81] Gorospe A, Nisay A, Dizon J R and Shin H S 2013 Delamination behaviour of GdBCO coated conductor tapes under transverse tension Physica C: Superconductivity 494 163-7

[82] Iwasa Y 2009 Case Studies in Superconducting Magnets - $2^{\text {nd }}$ edition: Springer)

[83] Kim S W, Sohn M H, Baik S K, Jo Y S, Seo M G, Lee E Y, Ko R K and Kwon Y K 2003 Soldered double pancake winding of high temperature superconducting tape Applied Superconductivity, IEEE Transactions on 13 1784-7

[84] Hazelton D W, Selvamanickam V, Duval J M, Larbalestier D C, Markiewicz W D, Weijers H W and Holtz R L 2009 Recent Developments in 2G HTS Coil Technology Applied Superconductivity, IEEE Transactions on $192218-22$

[85] Takao T, Iwamura T, Fukasawa Y, Minowa S, Sato H, Asano T, Ishiyama A, Kato J, Machi T, Nakao K and Shiohara Y 2007 Influence of Bending and Torsion Strains on Critical Currents in YBCO Coated Conductors Applied Superconductivity, IEEE Transactions on 17 3513-6

[86] Takao T, Sakabe R, Asano T, Mitsui S, Nakao K, Shiohara Y and Kashima N 2009 Degradation Due to Bending Fatigue Strain in YBCO Coated Conductors Applied Superconductivity, IEEE Transactions on 19 2988-90

[87] Markiewicz W D and Swenson C A 2010 Winding strain analysis for YBCO coated conductors Superconductor Science and Technology 23045017

[88] Shiroyanagi Y, Ghosh A K, Gupta R and Sampson W B 2011 The Construction and Testing of YBCO Pancake Coils for a High Field Solenoid Applied Superconductivity, IEEE Transactions on 21 1649-52

[89] Jeong H, Park H, Kim S, Park M, Yu I-K, Lee S, Park T, Sim K, Ha H-S, Oh S-S and Moon S-H 2012 DeLamination Characteristics of Coated Conductor for Conduction Cooled HTS Coil Applied Superconductivity, IEEE Transactions on 227700804

[90] Awaji S, Oguro H, Suwa T, Suzuki T, Watanabe K, Nishijima G, Hanai S, Marukawa K, Daibo M, Saito T, Sakamoto H, Inoue I, Miyoshi Y, Chaud X, Debray F and Tixador P 2013 Superconducting and Mechanical Properties of Impregnated REBCO Pancake Coils Under Large Hoop Stress Applied Superconductivity, IEEE Transactions on $\mathbf{2 3} 4600305$

[91] Uglietti D, Choi S, Matsumoto S and Kiyoshi T 2011 High current density in layer-wound coated conductorcoils Oficyna Wydawnicza Politechniki Wroclawskiej, Wroclaw, Poland 1219

[92] Mizuno K, Ogata M and Nagashima K 2013 An Innovative Superconducting Coil Fabrication Method with YBCO Coated Conductors Quarterly Report of RTRI 54 46-51

[93] Oomen M, Herkert W, Bayer D, Kummeth P, Nick W and Arndt T 2012 Manufacturing and test of 2G-HTS coils for rotating machines: Challenges, conductor requirements, realization Physica C: Superconductivity 482 111-8 
[94] Barth C, Bagrets N, Weiss K P, Bayer C M and Bast T 2013 Degradation free epoxy impregnation of REBCO coils and cables Superconductor Science and Technology 26055007

[95] Uglietti D, Choi S and Kiyoshi T 2010 Design and fabrication of layer-wound YBCO solenoids Physica C: Superconductivity 470 1749-51

[96] Badcock R A, Long N J, Mulholland M, Hellmann S, Wright A and Hamilton K A 2009 Progress in the Manufacture of Long Length YBCO Roebel Cables Applied Superconductivity, IEEE Transactions on 19 3244-7

[97] Weijers H W, Trociewitz U P, Markiewicz W D, Jiang J, Myers D, Hellstrom E E, Xu A, Jaroszynski J, Noyes P, Viouchkov Y and Larbalestier D C 2010 High Field Magnets With HTS Conductors Applied Superconductivity, IEEE Transactions on 20 576-82

[98] Bai H, Markiewicz W D, Lu J and Weijers H W 2013 Thermal Conductivity Test of YBCO Coated Conductor Tape Stacks Interleaved With Insulated Stainless Steel Tapes Applied Superconductivity, IEEE Transactions on 234600204

[99] Gupta R 2013 High Field HTS Solenoid for a Muon Collider Demonstrations, Challenges and Strategies International Conference on Magnet Technology MT23, Boston, US http://www.bnl.gov/magnets/staff/gupta/Talks/mt23/OrBB-02-gupta-mt23.pdf

[100] Gupta R, Anerella M, Ganetis G, Ghosh A, Kirk H, Palmer R, Plate S, Sampson W, Shiroyanagi Y, Wanderer P, Brandt B, Cline D, Garren A, Kolonko J, Scanlan R and Weggel R 2011 High Field HTS R\&D Solenoid for Muon Collider Applied Superconductivity, IEEE Transactions on 21 1884-7

[101] Yu M, Lombardo V, Lopes M L, Turrioni D, Zlobin A V, Flanagan G and Johnson R P 2011 Test of 1st YBCO helical solenoid double pancake model 2011 Particle Accelerator Conference 1118

[102] Park Y, Shin H-J, Kim Y-G, Oh Y and Lee H 2013 Effects of Melting Diffusion and Annealing in Oxygen on Superconducting Characteristics of GdBCO Coated Conductors: Preliminary Results Applied Superconductivity, IEEE Transactions on 236600804

[103] Park Y, Lee M, Ann H, Choi Y and Lee H 2013 A superconducting joint for GdBa2Cu3O7- $\delta$ coated conductors Nature Asia 6 e98

[104] http://www.superpower-inc.com/system/files/SP_Soldering+Instructions_2013FEC_v2.pdf

[105] Duckworth R C, Zhang Y, Gouge M J, Rey C M, van der Laan D C and Clickner C 2010 VOLTAGE DISTRIBUTION AND MECHANICAL STRENGTH IN SPLICE JOINTS MADE FROM ASMANUFACTURED YBCO COATED CONDUCTORS AIP Conference Proceedings 1219 370-9

[106] Kim Y, Bascunan J, Lecrevisse T, Hahn S, Voccio J, Park D K and Iwasa Y 2013 YBCO and Bi2223 Coils for High Field LTS/HTS NMR Magnets: HTS-HTS Joint Resistivity Ieee T Appl Supercon 236800704

[107] Chang K, Jo H, Kim Y, CheolAhn M and Ko T 2011 An Experimental Study on the Joint Methods Between Double Pancake Coils Using YBCO Coated Conductors Applied Superconductivity, IEEE Transactions on $213005-8$

[108] Chang K, Park D, Yang S, Jo H, Kim H, Yoon Y, Kim H, Lee H and Ko T 2010 Experimental Analysis of a Splice Method Between YBCO Coated Conductors on Various Bending Diameters Applied Superconductivity, IEEE Transactions on 20 1577-80

[109] Sugano M, Nakamura T, Shikimachi K, Hirano N and Nagaya S 2007 Stress Tolerance and Fracture Mechanism of Solder Joint of YBCO Coated Conductors Applied Superconductivity, IEEE Transactions on 17 3067-70

[110] Walsh R P, McRae D, Markiewicz W D, Lu J and Toplosky V J 2012 The 77-K Stress and Strain Dependence of the Critical Current of YBCO Coated Conductors and Lap Joints Applied Superconductivity, IEEE Transactions on 228400406

[111] Markiewicz D W, Larbalestier D C, Weijers H W, Voran A J, Pickard K W, Sheppard W R, Jaroszynski J, Xu A, Walsh R P, Lu J, Gavrilin A V and Noyes P D 2012 Design of a Superconducting 32 T Magnet With REBCO High Field Coils Applied Superconductivity, IEEE Transactions on 224300704

[112] Iwasa Y and Hahn S 2013 First-cut design of an all-superconducting 100-T direct current magnet Appl Phys Lett 103253507

[113] Nagaya S, Watanabe T, Tamada T, Naruse M, Kashima N, Katagiri T, Hirano N, Awaji S, Oguro H and Ishiyama A 2013 Development of High Strength Pancake Coil With Stress Controlling Structure by REBCO Coated Conductor Applied Superconductivity, IEEE Transactions on 234601204

[114] Nishijima G, Minegishi K, Awaji S, Watanabe K, Izumi T and Shiohara Y 2011 Hoop Stress Test of $\mathrm{GdBa}_{2} \mathrm{Cu}_{3} \mathrm{O}_{\mathrm{y}}$ Coated Conductor Applied Superconductivity, IEEE Transactions on 21 3094-7

[115] Grabovickic R, Lue J W, Gouge M J, Demko J A and Duckworth R C 2003 Measurements of temperature dependence of the stability and quench propagation of a 20-cm-long RABiTS Y-Ba-Cu-O tape Ieee T Appl Supercon 13 1726-30

[116] Trillaud F, Palanki H, Trociewitz U P, Thompson S H, Weijers H W and Schwartz J 2003 Normal zone propagation experiments on HTS composite conductors Cryogenics 43 271-9

[117] Wang X, Trociewitz U P and Schwartz J 2007 Near-adiabatic quench experiments on short YBa2Cu3O7delta coated conductors Journal of Applied Physics 101053904 
[118] Song H and Schwartz J 2009 Stability and Quench Behavior of $\mathrm{YBa}_{2} \mathrm{Cu}_{3} \mathrm{O}_{7-\mathrm{x}}$ Coated Conductor at $4.2 \mathrm{~K}$, Self-Field Applied Superconductivity, IEEE Transactions on 19 3735-43

[119] Badel A, Antognazza L, Therasse M, Abplanalp M, Schacherer C and Decroux M 2012 Hybrid model of quench propagation in coated conductors for fault current limiters Superconductor Science and Technology 25095015

[120] Chan W K and Schwartz J 2011 Three-Dimensional Micrometer-Scale Modeling of Quenching in HighAspect-Ratio YBa2Cu3O7-delta Coated Conductor Tapes-Part II: Influence of Geometric and Material Properties and Implications for Conductor Engineering and Magnet Design Ieee T Appl Supercon 21 362834

[121] Levin G A, Jones W A, Novak K A and Barnes P N 2011 The effects of superconductor-stabilizer interfacial resistance on quenching of a pancake coil made out of coated conductor Superconductor Science and Technology 24035015

[122] Lacroix C, Fournier-Lupien J H, McMeekin K and Sirois F 2013 Normal Zone Propagation Velocity in 2G HTS Coated Conductor With High Interfacial Resistance Applied Superconductivity, IEEE Transactions on 234701605

[123] Sumption M D, Majoros M, Susner M, Lyons D, Peng X, Clark C F, Lawless W N and Collings E W 2010 Thermal diffusion and quench propagation in YBCO pancake coils wound with $\mathrm{ZnO}$ and Mylar insulations Superconductor Science and Technology 23075004

[124] Ishmael S, Luo H, White M, Hunte F, Liu X T, Mandzy N, Muth J F, Naderi G, Ye L, Hunt A T and Schwartz J 2013 Enhanced Quench Propagation in $\mathrm{Bi}_{2} \mathrm{Sr}_{2} \mathrm{CaCu}_{2} \mathrm{O}_{\mathrm{x}}$ and $\mathrm{YBa}_{2} \mathrm{Cu}_{3} \mathrm{O}_{7-\mathrm{x}}$ Coils via a Nanoscale Doped-Titania-Based Thermally Conducting Electrical Insulator Applied Superconductivity, IEEE Transactions on $\mathbf{2 3} 7201311$

[125] Turenne M, Johnson R, Hunte F, Schwartz J and Song H 2009 Multi-purpose fiber optic sensors for high temperature superconducting magnets 23rd IEEE/NPSS Symposium on Fusion Engineering, 2009. SOFE 2009.

[126] Marchevsky M, Xie Y Y and Selvamanickam V 2010 Quench detection method for 2G HTS wire Superconductor Science and Technology 23034016

[127] Yoneda M, Nanato N, Aoki D, Kato T and Murase S 2011 Quench detection/protection of an HTS coil by AE signals Physica C: Superconductivity 471 1432-5

[128] Trillaud F, Ang I, Kim W-S, Lee H G, Iwasa Y and Voccio J P 2007 Protection and Quench Detection of YBCO Coils Results With Small Test Coil Assemblies Applied Superconductivity, IEEE Transactions on 17 2450-3

[129] Brechna H and Turowski P 1978 Training and degradation phenomena in superconducting magnets Proc. 6th Int. Conf. Magnet Tech. (MT-6) 597

[130] Tsukamoto O, Maguire J F, Bobrov E S and Iwasa Y 1981 Identification of Quench Origins in a Superconductor with Acoustic-Emission and Voltage Measurements Applied Physics Letters 39 172-4

[131] Ninomiya A, Arai K, Takano K, Ishigohka T, Kaiho K, Nakajima H, Tsuji H, Okuno K, Martovetsky N and Rodin I 2003 Diagnosis of ITER's large scale superconducting coils using acoustic emission techniques Ieee T Appl Supercon 13 1408-11

[132] Lee H, Kim H M, Jankowski J and Iwasa Y 2004 Detection of 'hot spots' in HTS coils and test samples with acoustic emission signals Ieee T Appl Supercon 14 1298-301

[133] Ishiyama A, Ueda H, Ando T, Naka H, Bamba S and Shiohara Y 2007 A Criterion for Determining Stabilizer Thickness of YBCO Coated Conductors Based on Coil Protection Applied Superconductivity, IEEE Transactions on 17 2430-3

[134] Uglietti D and Marinucci C 2012 Design of a Quench Protection System for a Coated Conductor Insert Coil Applied Superconductivity, IEEE Transactions on 224702704

[135] Dixon I R, Markiewicz W D, Murphy P, Painter T A and Powell A 2004 Quench detection and protection of the wide bore $900 \mathrm{MHz}$ NMR magnet at the National High Magnetic Field Laboratory Applied Superconductivity, IEEE Transactions on 14 1260-3

[136] Trociewitz U P, Czabaj B, Hong S, Huang Y, Knoll D C, Larbalestier D C, Markiewicz W D, Miao H, Meinesz M, Wang X and Schwartz J 2008 Quench studies on a layer-wound $\mathrm{Bi}_{2} \mathrm{Sr}_{2} \mathrm{CaCu}_{2} \mathrm{O}_{\mathrm{x}} / \mathrm{AgX}$ coil at 4.2 K Superconductor Science and Technology 21025015

[137] Markiewicz W D 2008 Protection of HTS Coils in the Limit of Zero Quench Propagation Velocity Applied Superconductivity, IEEE Transactions on 18 1333-6

[138] Noyes P D, Markiewicz W D, Voran A J, Sheppard W R, Pickard K W, Jarvis J B, Weijers H W and Gavrilin A V 2012 Protection Heater Development for REBCO Coils Applied Superconductivity, IEEE Transactions on 224704204

[139] Gömöry 1986 Small superconducting solenoid wound from non-insulated unstabilized multifilamentary $\mathrm{Nb}_{3} \mathrm{Sn}$ conductor Proc. IIR Conf., Recent achievements in cryoengineering Cryoprague 86 197-202

[140] Barkov L M, Bashtovoy N S, Bragin A V, Karpov S V, Okhapkin V S, Pivovarov S G, Ruban A A, Smakhtin V P and Snopkov I G 1999 Superconducting magnet system of the CMD-2 detector Applied Superconductivity, IEEE Transactions on 9 4644-7 
[141] Bragin A V, Barkov L M, Karpov S V, Okhapkin V S, Popov Y S, Ruban A A and Smakhtin V P 2008 Test Results of the Thin Superconducting Solenoid for the CMD-3 Detector Applied Superconductivity, IEEE Transactions on 18 399-402

[142] Hahn S, Park D, Bascunan J and Iwasa Y 2011 HTS Pancake Coils Without Turn-to-Turn Insulation Applied Superconductivity, IEEE Transactions on 21 1592-5

[143] Kim S B, Saitou A, Joo J H and Kadota T 2011 The normal-zone propagation properties of the noninsulated HTS coil in cryocooled operation Physica C: Superconductivity 471 1428-31

[144] Hahn S, Park D, Voccio J, Bascunan J and Iwasa Y 2012 No-Insulation (NI) HTS Inserts for $>1 \mathrm{GHz}$ LTS/HTS NMR Magnets Applied Superconductivity, IEEE Transactions on 224302405

[145] Hahn S, Kim Y, Ling J, Voccio J, Park D, Bascunan J, Shin H-J, Lee H and Iwasa Y 2013 No-Insulation Coil Under Time-Varying Condition: Magnetic Coupling With External Coil Applied Superconductivity, IEEE Transactions on $\mathbf{2 3} 4601705$

[146] Uglietti D 2013 Development of coated conductor coils without electrical insulation ICEC 24 - ICMC 2012 Proceedings 513

[147] Kim Y, Hahn S, Ling J, Kim K L, Song J, Voccio J, Bascunan J and Iwasa Y 2014 Partial-Insulation Winding Technique for NbTi Coils Applied Superconductivity, IEEE Transactions on $\mathbf{2 4} 4700505$

[148] Amemiya N and Akachi K 2008 Magnetic field generated by shielding current in high $\mathrm{T}_{\mathrm{c}}$ superconducting coils for NMR magnets Superconductor Science and Technology 21095001

[149] Decell R F 1963 Accurate Low Magnetic Field Measurements on Superconducting Coils Review of Scientific Instruments 34 914-7

[150] Mains E F 1968 High-Homogeneity Tape-Wound Superconducting Coil Journal of Applied Physics 39 2622

[151] Richards D B, Edwards L R, Cornforth C M and Legvold S 1970 Vibrating Sample Magnetic Image Effects in an $\mathrm{Nb}_{3} \mathrm{Sn}$ Solenoid Review of Scientific Instruments 41 647-9

[152] Gu C, Qu T and Han Z 2007 Measurement and Calculation of Residual Magnetic Field in a Bi2223/Ag Magnet Applied Superconductivity, IEEE Transactions on 17 2394-7

[153] Hahn S, Bascunan J, Kim W-S, Bobrov E, Lee H and Iwasa Y 2008 Field Mapping, NMR Lineshape, and Screening Currents Induced Field Analyses for Homogeneity Improvement in LTS/HTS NMR Magnets Applied Superconductivity, IEEE Transactions on 18 856-9

[154] Hahn S, Bascunan J, Lee H, Bobrov E, Kim W, Ahn M and Iwasa Y 2009 Operation and performance analyses of 350 and $700 \mathrm{MHz}$ low-/high-temperature superconductor nuclear magnetic resonance magnets: A march toward operating frequencies above $1 \mathrm{GHz}$ Journal of Applied Physics 105024501

[155] Ahn M, Yagai T, Hahn S, Ando R, Bascunan J and Iwasa Y 2009 Spatial and Temporal Variations of a Screening Current Induced Magnetic Field in a Double-Pancake HTS Insert of an LTS/HTS NMR Magnet Applied Superconductivity, IEEE Transactions on 19 2269-72

[156] Yanagisawa Y, Nakagome H, Uglietti D, Kiyoshi T, Hu R, Takematsu T, Takao T, Takahashi M and Maeda H 2010 Effect of YBCO-Coil Shape on the Screening Current-Induced Magnetic Field Intensity Applied Superconductivity, IEEE Transactions on 20 744-7

[157] Yanagisawa Y, Kominato Y, Nakagome H, Hu R, Takematsu T, Takao T, Uglietti D, Kiyoshi T, Takahashi M and Maeda H 2011 Magnitude of the Screening Field for YBCO Coils Applied Superconductivity, IEEE Transactions on 21 1640-3

[158] Otsuka A, Yanagisawa Y, Kiyoshi T, Maeda H, Nakagome H and Takeda M 2011 Evaluation of the Screening Current in a $1.3 \mathrm{GHz}$ NMR Magnet Using ReBCO Applied Superconductivity, IEEE Transactions on $212076-9$

[159] Uglietti D, Yanagisawa Y, Maeda H and Kiyoshi T 2010 Measurements of magnetic field induced by screening currents in YBCO solenoid coils Superconductor Science and Technology 23115002

[160] Iwasa Y, Hahn S, Voccio J, Park D K, Kim Y and Bascunan J 2013 Persistent-mode high-temperature superconductor shim coils: A design concept and experimental results of a prototype $\mathrm{Z} 1$ high-temperature superconductor shim Applied Physics Letters 10352607

[161] Yanagisawa Y, Nakagome H, Koyama Y, Hu R, Takao T, Hamada M, Kiyoshi T, Takahashi M and Maeda H 2009 Effect of current sweep reversal on the magnetic field stability for a Bi-2223 superconducting solenoid Physica C: Superconductivity 469 1996-9

[162] Kajikawa K and Funaki K 2011 A simple method to eliminate shielding currents for magnetization perpendicular to superconducting tapes wound into coils Superconductor Science and Technology 24 125005

[163] 2013 High Magnetic Field Science and Its Application in the United States: Current Status and Future Directions - National Academy of Science.

[164] Bascunan J, Hahn Y, Kim Y and Iwasa Y 2013 An 18.8-T/90-mm Bore All-HTS Insert (H800) for 1.3 GHz LTS/HTS NMR Magnet: Insert Design and Double Pancake Coil Fabrication Applied Superconductivity, IEEE Transactions on $\mathbf{2 4} 4300904$

[165] Bascunan J, Hahn S, Park D, Kim Y and Iwasa Y 2012 On the $600 \mathrm{MHz}$ HTS Insert for a $1.3 \mathrm{GHz}$ NMR Magnet Applied Superconductivity, IEEE Transactions on 224302104 
[166] Bascunan J, Hahn S, Kim Y and Iwasa Y 2013 A New High-Temperature Superconducting (HTS) 700MHz Insert Magnet for a 1.3-GHz LTS/HTS NMR Magnet Applied Superconductivity, IEEE Transactions on 234400304

[167] Otsuka A, Kiyoshi T and Takeda M 2010 A $1.3 \mathrm{GHz}$ NMR Magnet Design Under High Hoop Stress Condition Applied Superconductivity, IEEE Transactions on 20 596-9

[168] Kiyoshi T, Choi S, Matsumoto S, Zaitsu K, Hase T, Miyazaki T, Otsuka A, Yoshikawa M, Hamada M, Hosono M, Yanagisawa Y, Nakagome H, Takahashi M, Yamazaki T and Maeda H 2010 HTS-NMR: Present Status and Future Plan Applied Superconductivity, IEEE Transactions on 20 714-7

[169] Matsumoto S, Kiyoshi T, Otsuka A, Hamada M, Maeda H, Yanagisawa Y, Nakagome H and Suematsu H 2012 Generation of $24 \mathrm{~T}$ at $4.2 \mathrm{~K}$ using a layer-wound GdBCO insert coil with Nb3Sn and Nb-Ti external magnetic field coils Superconductor Science and Technology 25025017

[170] http://www.magnet.fsu.edu/usershub/publications/sciencehighlights/.

[171] Weijers H W, Markiewicz W D, Voran A J, Gundlach S R, Sheppard W R, Jarvis B, Johnson Z L, Noyes P D, Lu J, Kandel H, Bai H, Gavrilin A V, Viouchkov Y L, Larbalestier D C and Abraimov D V 2014 Progress in the Development of a superconducting $32 \mathrm{~T}$ magnet with REBCO high field coils Applied Superconductivity, IEEE Transactions on $\mathbf{2 4} 4301805$

[172] Awaji S, Ishihara R, Namba M, Nishijima G, Oguro H, Watanabe K, Shikimachi K, Hirano N and Nagaya S 2010 Upgrading Design to a 25 T Cryogen-Free Superconducting Magnet Based on Low Temperature and High Magnetic Field Properties of the Practical CVD Processed Coated Conductors Applied Superconductivity, IEEE Transactions on 20 592-5

[173] Awaji S, Watanabe K, Oguro H, Hanai S, Miyazaki H, Takahashi M, Ioka S, Sugimoto M, Tsubouchi H, Fujita S, Daibo M, Iijima Y and Kumakura H 2014 New 25 T Cryogen-Free Superconducting Magnet Project at Tohoku University Applied Superconductivity, IEEE Transactions on $\mathbf{2 4} 4302005$

[174] Koyanagi K, Ono M, Hanai S, Watanabe K, Awaji S, Hamajima T, Kiyoshi T and Kumakura H 2009 Design of a $30 \mathrm{~T}$ Superconducting Magnet Using a Coated Conductor Insert Applied Superconductivity, IEEE Transactions on 19 1617-20 